\title{
Chapitre VIII - Les personnels
}

\section{Maurice Wolkowitsch}

\section{(2) OpenEdition}

\section{Journals}

Édition électronique

URL : https://journals.openedition.org/rhcf/1321

DOI : 10.4000/rhcf.1321

Éditeur

Rails \& histoire

\section{Édition imprimée}

Date de publication : 10 février 2004

Pagination : 249-279

ISBN : 0996-9403

ISSN : 0996-9403

Référence électronique

Maurice Wolkowitsch, "Chapitre VIII - Les personnels », Revue d'histoire des chemins de fer [En ligne], 30 | 2004, mis en ligne le 21 août 2012, consulté le 22 avril 2022. URL : http://journals.openedition.org/ rhcf/1321 ; DOI : https://doi.org/10.4000/rhcf.1321 


\section{Chapitre VIII \\ Les personnels}

I es statistiques relatives aux chemins de fer secondaires font d'intérêt général : Administration centrale, Mouvement et Trafic, Matériel et Traction, Voie et Bâtiments. Les compagnies secondaires libérées de certaines obligations imposées aux grands réseaux se satisfont généralement d'un personnel dont l'éventail hiérarchique est simplifié.

Sauf mention contraire, les données brutes portent sur l'année 1911, les données comparatives et les taux de croissance sur la période 1901-1911. Les statistiques sont fournies pour chaque compagnie exploitant une ligne ou un réseau ; dans ce dernier cas, les chiffres visent l'ensemble du réseau ou chacune des lignes qui le composent. L'étude des catégories de personnel repose sur des échantillons représentatifs de compagnies, choisies en fonction du kilométrage exploité et des milieux desservis.

Le maniement des statistiques soulève certains problèmes. L'inconvénient du regroupement des fonctions est limité, s'il s'agit de l'exercice de mêmes fonctions (chef et sous-chef de gare), important s'il empêche de cerner les effectifs affectés à des tâches différentes (personnel de bureau et contremaitre travaillant dans les ateliers). L'emploi d'un personnel commun à plusieurs compagnies ou à toutes les lignes d'une même compagnie ne facilite pas la définition d'un indice fondé sur l'effectif employé pour un kilométrage donné ; nous avons retenu le nombre d'agents pour $10 \mathrm{~km}(\mathrm{Ag} / \mathrm{km})$, de préférence au kilomètre, pour rendre les données plus expressives; cette notion permet de comparer les politiques du personnel des compagnies.

\section{Évolution générale de l'emploi}

La première décennie du $\mathrm{x} \mathrm{x}^{\mathrm{e}}$ siècle est caractérisée par l'allongement spectaculaire des réseaux secondaires, entrainant l'augmentation des effectifs. Le taux de croissance de la longueur exploitée dépasse celui des embauches pour les TVM. Les effectifs et le kilométrage exploité des réseaux de TVM croissent davantage que ceux des CFIL; mais ces derniers retiennent encore $62,7 \%$ de la main-d'œuvre de cette branche d'activité (tabl. 46). 
Tableau 46. Kilométrage exploité et emploi

\begin{tabular}{|l|c|c|c|c|c|c|}
\hline \multirow{2}{*}{} & \multicolumn{3}{|c|}{ Longueur du réseau (km) } & \multicolumn{3}{c|}{ Nom bre d'em plois } \\
\cline { 2 - 7 } & 1901 & 1911 & $\begin{array}{c}\text { Evolution } \\
(\%)\end{array}$ & 1901 & 1911 & $\begin{array}{c}\text { Évolution } \\
(\%)\end{array}$ \\
\hline CFIL & 5935 & 9678 & +63 & 8908 & 15427 & +73 \\
\hline TVM & 3054 & 6392 & +109 & 4636 & 9184 & +98 \\
\hline Total & 8989 & 16070 & $+78,7$ & 13544 & 24611 & $+81,7$ \\
\hline
\end{tabular}

À côté d'un personnel permanent, les compagnies secondaires utilisent une main-d'œuvre payée à la journée, notamment pour l'entretien du matériel ; ces 2622 emplois précaires représentent 10,6 \% de la main-d'œuvre totale et sont particulièrement nombreux dans les compagnies de TVM, près de $14 \%$; le nombre de ces journaliers s'est beaucoup accru en dix ans, même si sa part a légèrement régressé.

L'emploi féminin progresse de $90 \%$, il représente $13,2 \%$ du total des agents. Ces femmes occupent des emplois de services dans les bureaux et des postes de chef de station. La rareté du gardiennage des voies à la traversée des routes les prive de l'opportunité d'exercer un métier qui leur est souvent confié sur les réseaux principaux (tabl. 47).

Tableau 47. Emploi précaire et emploi féminin

\begin{tabular}{|l|c|c|c|c|c|c|}
\hline \multirow{2}{*}{} & \multicolumn{2}{|c|}{ Emplois payés à la journée } & \multicolumn{3}{c|}{ Emplois fém inins } \\
\cline { 2 - 7 } & 1901 & 1911 & $\begin{array}{c}\text { Évolution } \\
(\%)\end{array}$ & 1901 & 1911 & $\begin{array}{c}\text { Évolution } \\
(\%)\end{array}$ \\
\hline CFIL & 1226 & 1357 & $+10,6$ & 1260 & 2154 & +70 \\
\hline TVM & 727 & 1265 & +74 & 444 & 1089 & +145 \\
\hline Total & 1953 & 2622 & +34 & 1704 & 3243 & +90 \\
\hline
\end{tabular}

La répartition des personnels entre les services est d'une grande stabilité : 5,4\% pour l'administration centrale, administrateurs inclus, $36,4 \%$ pour Mouvement et Trafic, 32,8 pour Voie et Bâtiments, 25,4 pour Matériel et Traction.

Le réseau ferré secondaire représente $39 \%$ du kilométrage exploité par les grandes compagnies (16 $070 \mathrm{~km}$ contre 40583 ) ; la proportion est de 6,9\% pour l'emploi (24 611 contre 349 721). Cette différence souligne la faiblesse de la main-d'œuvre nécessaire pour assurer le fonctionnement des compagnies secondaires. 


\section{L'organisation administrative des services}

\section{. L'administration centrale}

Mille deux cent quarante-cinq personnes forment la totalité des personnels des administrations centrales des compagnies secondaires, dont seulement le tiers pour celles des compagnies de TVM. On distingue, à la base, 83 concierges, garçons de bureau et gens de service, suivis à l'échelon suivant de 405 agents formant le personnel de bureau; le sommet de la hiérarchie comprend 757 administrateurs, directeurs, sousdirecteurs, membres des comités et secrétaires généraux.

Cette classification entretient la confusion entre des cadres supérieurs salariés et des administrateurs intéressés financièrement à la vie de l'entreprise. Les administrateurs sont souvent à l'origine de l'entreprise et de la construction d'une ligne; ils votent le budget, définissent la politique de développement du réseau, d'achat du matériel, de recrutement de la main d'œuvre; ils sont rémunérés ${ }^{1}$.

Beaucoup de compagnies, même grandes, se satisfont d'une administration centrale faiblement dotée en personnel : la Compagnie générale des chemins de fer vicinaux, avec $665 \mathrm{~km}$, a sept administrateurs, directeurs..., mais un garçon de bureau et un personnel de bureau limité à cinq personnes. L'échelon supérieur dominant les deux autres est le cas le plus fréquent, illustré par la Compagnie des tramways à vapeur du département de l'Aude, neuf et un. Les compagnies secondaires sont en quête d'économies, aussi les autres services suppléent-ils aux carences des administrations centrales en personnel de bureau.

La recherche d'économies passe aussi par l'utilisation en commun de personnel de plusieurs entreprises, sous des formes diverses; le rassemblement des sièges sociaux en un même lieu facilite cette politique $^{2}$. Certaines lignes secondaires, généralement à écartement normal, sont gérées par l'administration centrale d'un grand réseau : l'Est pour la ligne de Rambervillers à Charmes, l'État pour celle de Richelieu à Ligré-Rivière. Une compagnie secondaire peut aussi gérer plusieurs d'entre elles : la Compagnie des tramways du Loir-et-Cher gère la Compagnie des tramways de la Sarthe, la Compagnie du chemin de fer de Mamers à Saint-Calais et la ligne de Nantes à Legé de la Compagnie française des chemins de fer à voie étroite. Des solutions encore plus complexes sont utilisées pour économiser le personnel des administrations centrales : une série d'entreprises exploitant ensemble plus de $400 \mathrm{~km}$ disposent

1- Seule, la Société d'électricité du Mont-Dore et du chemin de fer du Capucin précise explicitement l'absence de rémunération.

2- cf. ci-dessus p. 109 et suiv. 
d'un ingénieur, d'un secrétaire général, de six employés de bureau et d'un garçon de bureau pour pallier l'absence de personnel propre à chaque ligne ou épauler celui qui y est affecté. Les administrateurs, directeurs... sont plus fréquemment représentés que le personnel d'exécution (tabl. 48).

Tableau 48. Personnel effecté aux lignes utilisant aussi le personnel commun signalé dans le texte

\begin{tabular}{|l|c|c|c|c|}
\hline \multicolumn{1}{|c|}{ Lignes } & Km & (a) & (b) & (c) \\
\hline Achiet à Bapaume et Marcoing & 32 & 11 & 7 & 1 \\
\hline Boisleux à Marquion & 26 & 7 & 0 & 0 \\
\hline Cambrai à Marquion & 20 & 0 & 0 & 0 \\
\hline Saint-Quentin à Vélu-Bertincourt & 52 & 12 & 0 & 0 \\
\hline Roisel à Hargicourt & 7 & $0(\mathrm{~d})$ & 0 & 0 \\
\hline La Fère à Crécy-Mortier & 22 & 10 & 0 & 0 \\
\hline $\begin{array}{l}\text { Berck à Rimeux et Gournay, Aire à } \\
\text { Fruges }\end{array}$ & 97 & 8 & 0 & 0 \\
\hline Lignes des Flandres & 43 & 6 & 0 & 0 \\
\hline Maubeuge à Villers-Sire-Nicole & 12 & 6 & 2 & 1 \\
\hline Ardres à Pont-d'Ardres & 6 & $0(\mathrm{e})$ & 0 & 0 \\
\hline Enghien à Montmorency & 10 & 0 & 0 & 0 \\
\hline Anvin à Calais & 94 & 6 & 0 & 0 \\
\hline
\end{tabular}

(a) Administrateurs, directeurs, sous-directeurs, membres des comités, secrétaires généraux. (b) Personnel de bureau. (c) Concierges, garçons de bureau, gens de service. (d) Mêmes administrateurs et directeurs que pour la ligne de Saint-Quentin à Vélu-Bertincourt. (e) Mêmes administrateurs et directeurs que pour la ligne de Calais à Anvin.

Les administrations centrales de deux compagnies de kilométrages voisins offrent des effectifs variant de 13 pour la Compagnie générale des chemins de fer vicinaux à 84 pour la Compagnie des chemins de fer départementaux ${ }^{3}$. Le personnel de l'administration centrale de la SGCFE relève statistiquement des effectifs des grands réseaux.

3- Rappelons que le réseau total de la Compagnie des chemins de fer départementaux comprend en outre des lignes affermées et des lignes d'intérêt général, ce qui justifie l'importance des effectifs de son administration centrale. 


\section{. Les divisions opérationnelles}

\section{$>$ Mouvement et Trafic (annexe 1)}

La division Mouvement et Trafic associe un service central au service des gares et stations et à celui des trains, respectivement $12 \%$, $68 \%$ et $20 \%$ de l'emploi dans cette division.

\section{Le service central de la division}

La hiérarchie du service central est calquée sur celle de l'administration centrale : un contingent inchangé en dix ans de 78 emplois subalternes, un personnel de bureau fort de 543 agents, en progrès de $60 \%, 261$ postes au sommet de directeurs, sous-directeurs, chefs et sous-chefs de l'exploitation et du mouvement, effectif de cadres concernés directement par le fonctionnement du chemin de fer et qui n'est pas gonflé comme dans l'administration centrale par la prise en compte des administrateurs. L'éventail hiérarchique est ici numériquement mieux respecté.

Service central de la division Mouvement et Trafic et administration centrale s'entraident dans de nombreuses compagnies, utilisant les mêmes locaux, les mêmes commis aux écritures, les mêmes gardiens. Une même politique de recherche d'économies conduit aux solutions évoquées à propos des administrations centrales. De petites entreprises exploitant moins de $50 \mathrm{~km}$ fonctionnent sans service central ${ }^{4}$. Les fonctions du service central de la division peuvent être confiées à une grande compagnie ou à une autre compagnie secondaire. Elles peuvent être regroupées pour une série de lignes exploitées ou non par une même compagnie ${ }^{5}$.

Sur les lignes de quelques dizaines de kilomètres, un service central limité au plus à trois agents suffit; il est exceptionnel de ne pas trouver un cadre. Au-delà de $100 \mathrm{~km}$, les entreprises disposent d'un service central étoffé avec un personnel d'exécution numériquement supérieur à l'encadrement ${ }^{6}$.

4- Le cas est plus fréquent pour les compagnies de TVM (Compagnie française du tramway à vapeur de Versailles à Maule, Compagnie du tramway d'Annecy à Thônes, Compagnie du chemin de fer de Taulignan à Grignan et Chamaret...) que de CFIL (Compagnie du chemin de fer de Berck-Plage à Paris-Plage...).

5- Le personnel de la Compagnie du chemin de fer de Hazebrouck à Merville (4 cadres, 9 employés de bureau, 2 garçons de service forment le service central) gère $200 \mathrm{~km}$ de lignes à voies normales ou étroites, exploitées par plusieurs compagnies relevant des deux régimes de CFIL : Hermes à Persan-Beaumont, Estrées-Saint-Denis à Froissy et Crèvecoeur, Milly à Formerie, Noyon à Lassigny et Guiscard, Méru à Labosse.

6- Société des chemins de fer de la Manche : 5 cadres sur 19 salariés ; Chemins de fer de l'Anjou : 5 cadres sur 28 salariés. 
Les trois principales compagnies ont inégalement doté leur service central : 30 agents pour la Compagnie générale des chemins de fer vicinaux, 12 pour la Compagnie des chemins de fer départementaux, 72 pour la SGCFE dont 29 cadres, 39 employés aux écritures et quatre garçons de bureau ; ces personnels peuvent être affectés au service central gérant une ligne (un cadre pour la liaison Gudmont à Rimaucourt) ou un réseau (cinq cadres, neuf employés, un garçon de bureau pour le réseau de la Gironde) ; la compagnie a concentré ses effectifs (dix cadres, vingt employés de bureau, trois garçons de bureau) dans le service central dirigeant son réseau du Centre rayonnant sur la Nièvre, le Cher et l'Allier.

\section{Les services des gares et trains}

Le service des gares emploie 6158 agents répartis en trois catégories. Au sommet, les chefs et sous-chefs gares, $52 \%$ des personnels de ce service, gèrent, parfois seuls, de nombreux points d'arrêt ; ils sont assistés dans les gares principales par les receveurs et facteurs enregistrants qui assument aussi la responsabilité de stations et haltes ; les chefs comptables sont affectés aux gares principales ou à celles où s'établissent les correspondances avec les grands réseaux. Troisième catégorie, les hommes d'équipe et ouvriers interviennent pour la manutention et les tâches les plus diverses ; eux aussi ne figurent que dans certaines gares.

Tous ces personnels affectés à chaque établissement ouvert au trafic sont indispensables au fonctionnement du système ferroviaire ; ils ne peuvent être communs à plusieurs lignes ; si plusieurs lignes utilisent une même gare, les statistiques indiquent généralement pour chacune d'entre elles le personnel qui y est affecté.

Le service des trains retient 1819 agents. Ce sont des « roulants ». On distingue d'une part les contrôleurs de route, chefs de train et sous-inspecteurs, d'autre part les conducteurs et gardes-freins. Le service des trains est plus consistant pour les TVM que pour les CFIL, peut-être par suite du plus grand nombre de points d'arrêt sans personnel, supposant la délivrance de billets dans le train.

\section{Les facteurs de variation des effectifs de la division Mouve- ment et Trafic}

Les effectifs de la division Mouvement et Trafic varient pour chaque entreprise en fonction du kilométrage exploité. Dans les entreprises exploitant moins de $100 \mathrm{~km}$, l'absence de certaines catégories de personnel est fréquente (annexe 1). La tendance logique est à la progression parallèle du kilométrage et de la main-d'œuvre, sans que puisse être reconnue une véritable proportion entre eux. 
Les lignes de 1 à $10 \mathrm{~km}$ offrent le nombre le plus élevé d'Ag/10 km, atteignant des sommets sur les liaisons entre des localités et les gares de grands réseaux (Cassel-gare à Cassel-ville, Beaucourt-Dasle à Beaucourt) ou sur des relations intra-urbaines (Rodez-gare à Rodez-palais de justice). La composition du personnel est simplifiée, avec généralement deux catégories sur trois pour le service des gares, une sur deux pour le service des trains (annexe 2); certains métiers sont interchangeables, comme chef de train et conducteur, la présence de l'un excluant celle de l'autre.

Le nombre d'Ag/10 km s'abaisse sur les lignes de 11 à $100 \mathrm{~km}$, s'établissant entre 2,4 et 9,5 contre 4,4 à 50 dans le cas précédent. Des situations contradictoires apparaissent : deux lignes d'une même longueur, à 1 ou $2 \mathrm{~km}$ près, offrent soit des taux voisins, comme Don à Fromelles et Amplepluis à Saint-Vincent-de-Reins, soit des taux allant du simple au double, comme Pithiviers à Toury et Pontarlier à Mouthe. Ces compagnies emploient rarement des conducteurs et gardes-freins ; toutes utilisent des chefs et sous-chefs de gares et dans deux tiers des cas étudiés quelques agents des autres catégories du service des gares (annexe 2).

Les compagnies exploitant plus de $100 \mathrm{~km}$ comptent, sauf exception, entre 3,4 et $5,3 \mathrm{Ag} / 10 \mathrm{~km}$, chiffres encadrant la moyenne nationale de 4,2. Toutes les compagnies font appel aux trois catégories d'agents du service des gares, sauf le réseau de tramways de la Compagnie des chemins de fer des Charentes qui n'emploie ni receveur, ni facteur enregistrant. Plusieurs entreprises se contentent d'une seule catégorie d'agents pour le service des trains. Le nombre d'Ag/10 km est proche de la moyenne pour les trois grandes compagnies dépassant $600 \mathrm{~km}$ : SGCFE, 4,4 avec un total de 794 employés ; la Compagnie générale des chemins de fer vicinaux, 4 et 269 ; la Compagnie générale des chemins de fer départementaux, 5,1 et 332 .

Hors la longueur des lignes ou réseaux, d'autres facteurs pèsent sur le nombre d'Ag/10 km. Le régime d'exploitation en est un : le taux moyen s'élève à 8,2 pour les lignes régies par la loi de 1865 , il n'est que de 4,7 pour celles régies par la loi de 1880 ou dépendant des TVM. Ce facteur se combine avec l'écartement des voies, le taux moyen est fort pour les voies à écartement normal, 6,7 contre 4,5 pour les lignes à voies étroites ; or le réseau de 1865 comporte un fort pourcentage de liaisons à voie normale. Ces résultats sont confirmés par les taux observés sur des lignes de longueurs voisines relevant de deux compagnies utilisant les deux types d'écartement (tabl. 49). 
Tableau 49. Nombre d'Ag/10 km sur les lignes de deux compagnies n'offrant pas le même type d'écartement

\begin{tabular}{|l|l|c|c|c|}
\hline \multicolumn{1}{|c|}{ Compagnie } & \multicolumn{1}{|c|}{ Ligne } & Km & Écartement & $\mathbf{A g / 1 0 ~} \mathbf{~ k m}$ \\
\hline \multirow{2}{*}{ Michon } & Don à Fromelles & 18 & Normal & 4,4 \\
\cline { 2 - 5 } & $\begin{array}{l}\text { Hondschoote à } \\
\text { Bray-Dunes }\end{array}$ & 15 & Étroit & 2,6 \\
\hline $\begin{array}{l}\text { Compagnie des chemins } \\
\text { de fer de la Limagne }\end{array}$ & Gerzat à Maringues & 20 & Normal & 6 \\
\cline { 2 - 6 } & Riom à Volvic & 18 & Étroit & 2,2 \\
\hline
\end{tabular}

\section{$>$ Matériel et Traction (annexe 2)}

L'effectif de ce service, 6264 emplois, progresse de $81 \%$. 49,4\% des agents sont des roulants, mécaniciens, chauffeurs et wattmen, 41,2\% des ouvriers et des gens de service à l'année ou à la journée ; restent 9,4 \% partagés à égalité entre ingénieurs, chef de dépôt, sous-chef d'atelier et personnel de bureau des dépôts.

L'ouverture d'une section de ligne nouvelle ou complément d'un réseau préexistant exigeait le recrutement des responsables des gares et stations mais, pour l'entretien du matériel et les services des roulants, cela permettait une utilisation plus rationnelle de la main-d'œuvre. Le matériel, sauf celui des compagnies régies par la loi de 1865, est souvent récent, notamment pour les TVM. Si aucune entreprise ne pouvait fonctionner sans au moins un mécanicien ${ }^{7}$, beaucoup limitaient les dépenses d'entretien du matériel : sur un échantillon de 60 entreprises, $55 \%$ sont sans personnel de bureau des dépôts, $20 \%$ sans cadre, $10 \%$ sans personnel d'entretien. Le nombreux personnel recruté à la journée permettait d'adapter le volume de la main-d'œuvre au travail à effectuer. Certains mécaniciens étaient sans doute formés à l'entretien de leur machine. Bien des compagnies disposaient d'un parc réduit de wagons, voitures, engins de traction ne justifiant pas l'existence d'ateliers et dépôts dotés d'une main-d'œuvre spécialisée comme sur les grands réseaux. Ce problème de l'entretien des matériels demanderait des recherches approfondies.

7- L'entreprise exploitant les $5 \mathrm{~km}$ de la ligne de TVM de Laboutarié à Réalmont emploie un mécanicien, seul agent du service Matériel et Traction. 
L’effectif dépend du kilométrage exploité ; mais des différences appréciables peuvent tout de même apparaitre : la Compagnie de l'Hérault et le réseau de la Compagnie des tramways à vapeur des Deux-Sèvres, de longueurs comparables, emploient respectivement 161 et 51 personnes (annexe 2).

Le nombre d'Ag/10km, emplois non permanents inclus, est de 4,3 pour les TVM, 5,2 pour les CFIL loi de 1865 , mais de seulement 3,2 pour les CFIL loi de 1880 où domine la voie étroite. L'éventail se resserre à mesure de l'augmentation de la distance : 2 à $50 \mathrm{Ag} / 10 \mathrm{~km}$ pour les lignes de 1 à $10 \mathrm{~km}, 1,1$ à 12 pour les lignes de 11 à $100 \mathrm{~km}$, 2,5 à 7,7 au-delà de $100 \mathrm{~km}$ exploités. Ces taux se retrouvent pour les principales compagnies; $5 \mathrm{Ag} / 10 \mathrm{~km}$ est atteint pour les lignes à voie normale de la Compagnie générale des chemins de fer départementaux. La SGCFE, avec 3,5 Ag/10 km, bénéficie de l'extension de son réseau à voie étroite et de la possibilité d'utiliser le même personnel pour l'entretien des matériels des réseaux du Centre.

La composition des personnels des entreprises dépend de leur taille (annexe 2). Jusqu'à $100 \mathrm{~km}$, l'emploi de personnel de bureau des dépôts est exceptionnel ; plus surprenant, la moitié d'entre elles se passe de personnel ouvrier pour l'entretien du matériel ; en revanche, beaucoup ont un ingénieur ou un chef d'atelier. Les compagnies exploitant un réseau étendu se dotent de toutes les catégories de personnel, s'assurant un fonctionnement plus satisfaisant; les autres souffrent d'une irrégularité des services peut-être liée au manque d'entretien du matériel et à un effectif de mécaniciens insuffisant pour faire face à l'imprévu.

$>$ Voie et Bâtiments (annexe 3)

L'effectif de cette division s'élève de 4225 à 8081 agents (+ $91 \%$ ). Les agents de la surveillance et de l'entretien des voies, hommes d'équipe, ouvriers et gens de service forment les gros bataillons $(94,5 \%)$, réduisant les cadres à la portion congrue $(1,5 \%)$. Les catégories intermédiaires englobent des métiers divers : personnel de bureau peu nombreux, conducteurs et piqueurs, ces deux derniers étant responsables des travaux sur la voie.

L’entretien de la voie étant une nécessité quotidienne pour assurer la sécurité, un allongement significatif du kilométrage exploité entrainait un recrutement de main-d'œuvre; ce kilométrage était un facteur déterminant du volume de main-d'œuvre nécessaire à une entreprise ; ce personnel était affecté à une section de ligne. Cela n'exclut pas l'interférence avec d'autres facteurs rencontrés pour d'autres métiers du rail, le régime d'exploitation et l'écartement. L'exemple de deux lignes de cinq $\mathrm{km}$ est démonstratif : la division Voie et Bâtiments occupe six 
personnes sur la ligne de CFIL à écartement normal, régime de 1880, entre Aulnoye et Pont-sur-Sambre, une seule sur celle de TVM de Laboutarié à Réalmont (annexe 3).

La composition du personnel et l'éventail hiérarchique de cette division sont particulièrement simplifiés (annexe 3). $100 \%$ des compagnies étudiées jusqu'à $10 \mathrm{~km}, 85 \%$ de 11 à 100 , encore $42 \%$ au-delà de 100 et même la Compagnie générale des chemins de fer départementaux avec ses $666 \mathrm{~km}$ n'emploient aucun cadre. Pour les compagnies qui en comptent dans leur rang, souvent un seul, la statistique ne permet pas une approche satisfaisante ; elle mêle des métiers aussi divers qu'architecte et ingénieur ou des niveaux de compétence comme ingénieur et chef de section. Jusqu'à $100 \mathrm{~km}$, deux tiers des entreprises n'ont pas de conducteur de chantier et la surveillance quotidienne de la voie est dévolue à des agents de faible qualification.

Le nombre d'Ag/10 km dépend des facteurs précédemment évoqués, les compagnies principales enregistrant des taux moyens qui ne les singularisent pas par rapport aux autres.

\section{La politique sociale des compagnies de chemins de fer secondaires}

Les compagnies secondaires, étant très nombreuses, n’offrent pas les convergences observées dans les politiques du personnel des grands réseaux. Si la définition des métiers est identique, l'absence d'une grille salariale et d'un statut communs à toutes les entreprises de chemins de fer secondaires relativise la notion d'éventail hiérarchique ; la formule «à travail égal, salaire égal » ne caractérise pas cette activité.

Seulement une quarantaine d'entreprises de CFIL sont signalées comme disposant d'institutions de retraites et de secours : les caisses correspondantes sont alimentées par des prélèvements sur les salaires et par des allocations des compagnies. Les parts respectives varient dans de fortes proportions : les compagnies fournissent $100 \%$ des versements pour les caisses de secours des compagnies de l'Est de Lyon, du Cambrésis, d'Anvin à Calais, seulement $9 \%$ pour la Compagnie de chemins de fer du Morbihan. Les mêmes pourcentages ne se retrouvent pas systématiquement pour les deux caisses : ainsi, pour la caisse de retraite, la Compagnie du Morbihan fournit $50 \%$, celle des Ardennes $60 \%$, mais cette dernière verse $24 \%$ des recettes de la caisse de secours. Les exemples pourraient être multipliés et offrir la plus grande variété de cas de figures. 
Chaque entreprise définit son propre régime de retraite et de secours, mais on relève des cas particuliers. Les compagnies exploitant conjointement des lignes d'intérêt général et d'intérêt local (État, Est, SGCFE, CFD, Médoc, Sud-France) appliquent à l'ensemble de leur personnel les règles en usage sur les lignes d’intérêt général. Les personnels des compagnies étroitement associées ou contrôlées par des grands réseaux sont directement affiliés aux caisses de retraite et de secours de ces réseaux : Nord (Pont-de-la-Deule à Pont-à-Marcq...), Midi (Landes, Born et Marensin...) ; l'affiliation à la caisse de retraite d'une société industrielle utilisatrice de la ligne intervient pour les agents de la ligne de Rouen au Petit-Quevilly (Société Malétra). Des systèmes d'assurance de capitaux différés (Tramways de la Sarthe) ou d'assurance accidents gérés par les agents (Marlieux à Châtillon-sous-Chalaronne) existent également.

Les institutions de secours interviennent surtout en faveur des agents blessés ou, en cas de décès, de leur famille : la moyenne annuelle entre 1901 et 1910 a été de six morts et 48 blessés ${ }^{8}$.

Ces personnels ne bénéficient ni des salaires, ni des conditions de travail des cheminots des grandes compagnies. Les mesures adoptées par ces dernières sont transposées avec retard et moins de rigueur dans les compagnies secondaires, qui peuvent légalement pratiquer chacune leur propre politique. Le personnel est souvent peu formé à ses tâches et sous-qualifié : des trois obligations réglementaires de recruter des Français, sachant lire et écrire, seule la première est respectée ; encore en 1919, on apprend le règlement par cœur à des mécaniciens qui ne savent pas lire ! La productivité est très inégale : le chargement en charbon d'un même type de locomotive demande, suivant les équipes, de 8 à 30 minutes. Les bons éléments cherchent un emploi mieux payé et quittent au plus vite ces compagnies, surtout celles qui lésinent sur les salaires. Les conditions de travail sur le double plan de la durée de la journée de travail et des temps de repos ont évolué entre 1900 et 1940.

\section{. Temps de travail, temps de repos}

En 1900, les sédentaires travaillent en principe treize heures, les roulants douze ; certains mécaniciens travaillent seize heures lorsqu'un aller-retour sur une ligne longue imposait cette amplitude pour permettre le retour à domicile. Dès 1903, des grèves se produisent pour demander la journée de dix heures ; en 1906, la journée de huit heures devient la revendication permanente jusqu'à sa satisfaction accordée par la loi du 23 avril 1919. Son application entraîne une réduction des

8- Les agents tués ou blessés pendant cette période l'ont été pour 14 et 211 d'entre eux du fait de l'exploitation, pour 44 et 268 «par imprudence». 
horaires d'ouverture des gares au public ; elle s'accompagne sur le service des voies de la suppression des pauses casse-croûte et de la récupération des jours fériés; elle oblige à des embauches de roulants et à l'établissement de roulements exigeant davantage de découchés. Le personnel habitant à plus de $2 \mathrm{~km}$ de son lieu de travail a droit à $15 \mathrm{mi}-$ nutes décomptées dans le temps de travail.

En 1899, les grandes compagnies portent le temps de repos des mécaniciens de 24 à 30 heures tous les dix jours pour ceux qui découchent, tous les quinze jours pour les autres. Les compagnies secondaires appliquent la mesure en 1903 et seulement aux roulants ayant à découcher et cela, tous les quinze jours. Les roulements des mécaniciens pour des circulations à faible distance permettant fréquemment le retour à domicile, la majeure partie des personnels n'était pas concernée ; c'est un bon exemple de la façon dont les compagnies secondaires limitaient l'effet des mesures prises par les grandes compagnies.

En 1907, le principe de la journée hebdomadaire de repos est reconnu, mais sous astreinte, les agents devant rester à leur domicile. Certaines compagnies octroient des congés à leur personnel, alertées par les ingénieurs des Ponts et Chaussées sur la nécessaire réduction du temps de travail pour assurer la sécurité et par souci d'humanité. Le Blanc-Argent décide en 1914 d'accorder douze jours de congé annuel, plus trois jours par mois, ces derniers réservés à certains emplois. Le congé est porté à 21 jours en 1931. Les gardes-barrières bénéficient d'avantages en nature, le logement et un lopin pour cultiver des légumes : sur des lignes où, deux sens confondus, les mouvements sont le plus souvent de quatre à six trains par jour, ces agents sont considérés comme effectuant moins d'une heure de service pour la compagnie ; ils ont deux jours de repos par an et reçoivent des indemnités annuelles variant suivant les trafics ferroviaire et routier entre 30 et $150 \mathrm{~F}$ par an. Une mesure sociale d'importance est prise en 1923, avec retard sur les grands réseaux, la création obligatoire d'une caisse de retraite ; l'affiliation reposait sur le volontariat; les cotisations variaient suivant les départements de 2 à $4 \%$ du salaire, les compagnies donnaient autant, plus dans de rares cas, mais toujours un très faible pourcentage sur la recette brute. Cette mesure uniformisait la situation des personnels visà-vis de la retraite, chacun restant cependant libre de s'affilier ou non à la caisse.

\section{. Les politiques salariales (annexe 4)}

Les différences de salaires doivent être observées entre compagnies et au sein des compagnies. La comparaison entre onze compagnies est significative (tabl. 50). 
Tableau 50. Différences de salaires entre 11 compagnies (1911)

\begin{tabular}{|l|c|c|c|c|c|c|}
\hline \multirow{2}{*}{} & \multicolumn{3}{|c|}{ Personnel d'exécution } & \multicolumn{2}{c|}{ Personnel d'encadrement } \\
\cline { 2 - 3 } & $\begin{array}{c}\text { Compagnies } \\
\text { accordant les } \\
\text { salaires }\end{array}$ & Différence & \multicolumn{2}{c|}{$\begin{array}{c}\text { Compagnies } \\
\text { accordant les } \\
\text { salaires }\end{array}$} & \multirow{2}{*}{$\begin{array}{c}\text { Différence } \\
\%\end{array}$} \\
\cline { 2 - 3 } & $\begin{array}{c}\text { les moins } \\
\text { élevés }\end{array}$ & $\begin{array}{c}\text { les plus } \\
\text { élevés }\end{array}$ & & $\begin{array}{c}\text { les moins } \\
\text { élevés }\end{array}$ & $\begin{array}{c}\text { les plus } \\
\text { élevés }\end{array}$ & \\
\hline $\begin{array}{l}\text { Salaire } \\
\text { d'embauche }\end{array}$ & $6000 \mathrm{~F}$ & $7700 \mathrm{~F}$ & +28 & $8200 \mathrm{~F}$ & $17951 \mathrm{~F}$ & +118 \\
\hline $\begin{array}{l}\text { Salaire de fin } \\
\text { de carrière }\end{array}$ & $8568 \mathrm{~F}$ & $9010 \mathrm{~F}$ & $+5,1$ & $12622 \mathrm{~F}$ & $28000 \mathrm{~F}$ & +121 \\
\hline Progression & $+43 \%$ & $+14 \%$ & & $+54 \%$ & $+56 \%$ & \\
\hline
\end{tabular}

À l'embauche, on le voit, la différence entre les compagnies généreuses et celles qui le sont moins est de $28 \%$ pour le personnel d'exécution, de $118 \%$ pour le personnel d'encadrement, ces taux sont respectivement en fin de carrière de $5,1 \%$ et $121 \%$. Les taux très élevés, de l'ordre de $120 \%$ pour l'encadrement, s'expliquent par des différences sensibles de qualification (tabl. 50). Le nombre de compagnies pratiquant un niveau donné de salaire donne une idée de leur pratique sociale. L'échantillon choisi de onze compagnies est limité ; il comprend cependant deux des principales compagnies, une régie, mais pas de représentation des plus petites entreprises. La SGCFE (7 $700 \mathrm{~F}$ ) est la compagnie la plus généreuse à l'embauche pour le personnel d'exécution, la Régie départementale des tramways du Loir-et-Cher verse les salaires les plus bas et les plus élevés (tabl. 51).

Tableau 51. Nombre de compagnies versant des salaires d'un niveau donné (sur 11 compagnies, 1911)

\begin{tabular}{|c|c|c|c|c|c|}
\hline & \multicolumn{2}{|c|}{$\begin{array}{l}\text { Personnel } \\
\text { d'exécution }\end{array}$} & & \multicolumn{2}{|c|}{$\begin{array}{c}\text { Personnel } \\
\text { d'encadrement }\end{array}$} \\
\hline & $\begin{array}{c}\text { Salaire le } \\
\text { moins } \\
\text { élevé }\end{array}$ & $\begin{array}{l}\text { Salaire le } \\
\text { plus élevé }\end{array}$ & & $\begin{array}{c}\text { Salaire le } \\
\text { moins } \\
\text { élevé }\end{array}$ & $\begin{array}{l}\text { Salaire le } \\
\text { plus élevé }\end{array}$ \\
\hline 6000 à $7000 \mathrm{~F}$ & 7 & 0 & $<10000 \mathrm{~F}$ & 3 & 0 \\
\hline 7001 à $8000 \mathrm{~F}$ & 4 & 6 & 10000 à $15000 \mathrm{~F}$ & 5 & 5 \\
\hline 8001 à $9000 \mathrm{~F}$ & 0 & 4 & 15001 à $20000 \mathrm{~F}$ & 3 & 3 \\
\hline$>9000 \mathrm{~F}$ & 0 & 1 & $>20000 \mathrm{~F}$ & 0 & 3 \\
\hline
\end{tabular}


L'éventail des salaires varie sensiblement au sein de chaque entreprise ; pour le personnel d'exécution, la différence s'échelonne entre 700 et $1500 \mathrm{~F}$, pour les cadres entre 1150 et 12000 F. Á l'exception des Tramways de la Vienne, la différence entre les salaires de base et les salaires de fin de carrière des deux catégories de personnel est nettement plus forte pour les cadres. La comparaison entre la SGCFE et les CFD indique deux politiques en matière de niveau et d'éventail hiérarchique des salaires $^{9}$ (tabl. 52).

Tableau 52. Éventail salarial au sein des entreprises en francs - Différences en pourcentage et en francs entre les salaires les plus et les moins élevés

\begin{tabular}{|c|c|c|c|c|c|c|c|c|}
\hline \multirow{3}{*}{$\begin{array}{l}\text { Compagnies } \\
\text { SGCFE }\end{array}$} & \multirow{2}{*}{\multicolumn{2}{|c|}{$\begin{array}{l}\text { Personnel } \\
\text { d'exécution }\end{array}$}} & \multicolumn{2}{|c|}{ Différence } & \multirow{2}{*}{\multicolumn{2}{|c|}{$\begin{array}{c}\text { Personnel } \\
\text { d'encadrement }\end{array}$}} & \multicolumn{2}{|c|}{ Différence } \\
\hline & & & \multirow{2}{*}{\begin{tabular}{r|}
$\%$ \\
17
\end{tabular}} & \multirow{2}{*}{\begin{tabular}{|c|}
$\mathbf{F}$ \\
1310
\end{tabular}} & & & \multirow{2}{*}{$\begin{array}{l}\% \\
49\end{array}$} & \multirow{2}{*}{$\begin{array}{c}\mathbf{F} \\
6855 \mathrm{~F}\end{array}$} \\
\hline & $7700 \mathrm{~F}$ & $9010 \mathrm{~F}$ & & & $13905 \mathrm{~F}$ & $20760 \mathrm{~F}$ & & \\
\hline CFD & $6940 \mathrm{~F}$ & $7800 \mathrm{~F}$ & 12 & 860 & $11280 \mathrm{~F}$ & $13380 \mathrm{~F}$ & 19 & $2100 \mathrm{~F}$ \\
\hline Le Blanc-Argent & $7130 \mathrm{~F}$ & $8470 \mathrm{~F}$ & 18 & 1340 & $17955 \mathrm{~F}$ & $27200 \mathrm{~F}$ & 51 & $9245 \mathrm{~F}$ \\
\hline $\begin{array}{l}\text { Tramw ays de } \\
\text { l'Indre }\end{array}$ & $6660 \mathrm{~F}$ & $7992 \mathrm{~F}$ & 20 & 1332 & $9990 \mathrm{~F}$ & $13320 \mathrm{~F}$ & 33 & $3330 \mathrm{~F}$ \\
\hline $\begin{array}{l}\text { Tramw ays de la } \\
\text { Vienne }\end{array}$ & $7081 \mathrm{~F}$ & $8301 \mathrm{~F}$ & 17 & 1220 & $15201 \mathrm{~F}$ & $16351 \mathrm{~F}$ & 7 & $1150 \mathrm{~F}$ \\
\hline $\begin{array}{l}\text { Tramw ays des } \\
\text { Deux-Sèvres }\end{array}$ & $6832 \mathrm{~F}$ & $7532 \mathrm{~F}$ & 10 & 700 & $10382 \mathrm{~F}$ & $12622 \mathrm{~F}$ & 21 & $1790 \mathrm{~F}$ \\
\hline $\begin{array}{l}\text { Chemin de fer de } \\
\text { la Haute-Vienne }\end{array}$ & $7300 \mathrm{~F}$ & $8568 \mathrm{~F}$ & 17 & 1268 & $11640 \mathrm{~F}$ & $16440 \mathrm{~F}$ & 41 & $4800 \mathrm{~F}$ \\
\hline $\begin{array}{l}\text { Tramw ays de la } \\
\text { Sarthe }\end{array}$ & $6600 \mathrm{~F}$ & $7710 \mathrm{~F}$ & 17 & 1100 & $10980 \mathrm{~F}$ & 15480 & 41 & $4500 \mathrm{~F}$ \\
\hline $\begin{array}{l}\text { Tramw ays du } \\
\text { Loiret }\end{array}$ & $6850 \mathrm{~F}$ & $8140 \mathrm{~F}$ & 19 & 1290 & $9680 \mathrm{~F}$ & $14900 \mathrm{~F}$ & 54 & $5220 \mathrm{~F}$ \\
\hline \multicolumn{9}{|l|}{ Loir-et-Cher : } \\
\hline $\begin{array}{l}\text { - Régie des } \\
\text { Transports à } \\
\text { vapeur }\end{array}$ & $6000 \mathrm{~F}$ & $7500 \mathrm{~F}$ & 25 & 1500 & $16000 \mathrm{~F}$ & $28000 \mathrm{~F}$ & 75 & $12000 \mathrm{~F}$ \\
\hline $\begin{array}{l}\text { - Tramw ays } \\
\text { électriques }\end{array}$ & $6000 \mathrm{~F}$ & $7500 \mathrm{~F}$ & 25 & 1500 & $8200 \mathrm{~F}$ & $13570 \mathrm{~F}$ & 68 & $5730 \mathrm{~F}$ \\
\hline
\end{tabular}

9- L'étude des conditions de travail et des salaires doit beaucoup à l'analyse présentée par B. Moreau, Le Tacot, petit train berrichon, 2 tomes, Châteauroux, 1988, 122 p. et Issoudun, 1989, 176 p., p. 121-129. 
La recherche constante d'économies se traduit d'abord dans la faiblesse de l'encadrement, sensible, on l'a dit, dans les divisions Matériel et Traction, Voie et Bâtiments. Elle se traduit aussi dans les glissements de fonctions au sein de chaque service. Le service des gares illustre parfaitement cette constatation. Receveur, facteur enregistrant, à la limite homme d'équipe se voient confier des responsabilités tenues ailleurs par des chefs ou sous-chefs de gare. Chefs et sous-chefs de gare représentent plus de $85 \%$ du personnel du service des gares des Compagnies de chemins de fer des Ardennes, des Charentes, de Reims et de sa banlieue, mais seulement 3,5\% pour les Chemins de fer de la Sarthe et 7,5\% pour les Tramways du Loir-et-Cher; dans ces derniers cas, les receveurs et facteurs enregistrants fournissent respectivement 83 et $70 \%$ du service des gares. La part dévolue aux hommes d'équipe varie entre $3 \%$ sur le réseau de la Drôme et $53 \%$ sur celui de l'Hérault.

Le nombre d'Ag/10 km pour l'ensemble des personnels, administration centrale incluse, varie évidemment en fonction des facteurs révélés par l'analyse par service. Les lignes à voie normale régies par la loi de 1865 se singularisent par un taux élevé de 28, alors qu'il se situe entre 14 et 15 pour les autres CFIL et les TVM. Le milieu naturel est un facteur à ne pas négliger: le taux dépasse 18 sur une série de lignes situées dans les zones montagneuses, il ne dépasse pas treize et s'abaisse même jusqu'à sept sur des lignes de plaines $^{10}$.

\section{Comparaison entre les personnels des grandes compa- gnies et des compagnies secondaires}

L'administration centrale occupe une part beaucoup plus importante du personnel dans les compagnies secondaires $(5,3 \%)$ que dans les grands réseaux $(0,9 \%)$. Cette situation est liée à la dispersion des compagnies secondaires, chacune avec son administration centrale, face à la concentration des grands réseaux qui limite le nombre des dirigeants, 245 administrateurs et directeurs pour les grands réseaux, 757 pour les compagnies secondaires ; mais les premiers disposent d'un fort contingent de personnels de bureau et de service (2 996 agents), les seconds d'effectifs restreints (550 agents). Si on poursuit l'analyse, on constate que les dirigeants forment seulement $7 \%$ de l'administration centrale des grands réseaux, contre $62 \%$ pour les compagnies secondaires. La division Mouvement et Trafic domine l'ensemble des réseaux

10- Chamonix au Montenvers : 96 ; Pierrefitte-Nestalas à Cauteret : 32,3 ; Remiremont à Gérardmer : 20,3 ; vallée de Celles : 19,5; Pontarlier à Mouthe : 18,3; lignes des Flandres : 13 ; Pithiviers à Toury : 12,5; Challans à Fromentine : 10,4; Chemins de fer des Landes : 7,1. 
ferrés ; son importance est plus marquée pour les grands réseaux où l'action commerciale s'était développée avec des agents spécialisés et où la surveillance des circulations exigeait un personnel rare ou absent des compagnies secondaires (tabl. 53).

Tableau 53. Comparaison entre les personnels des grands réseaux et les compagnies secondaires

\begin{tabular}{|l|c|c|c|c|c|}
\hline & $\begin{array}{c}\text { Administration } \\
\text { centrale }\end{array}$ & $\begin{array}{c}\text { Mouvement } \\
\text { et Trafic }\end{array}$ & $\begin{array}{c}\text { Matériel et } \\
\text { Traction }\end{array}$ & $\begin{array}{c}\text { Voie et } \\
\text { Bâtiments }\end{array}$ & Total \\
\cline { 2 - 6 } & \multicolumn{5}{|c|}{ 1- Effectifs (unité) } \\
\hline $\begin{array}{l}\text { Chemins de fer } \\
\text { secondaires }\end{array}$ & 1307 & 8959 & 6264 & 8081 & 24611 \\
\hline Grands réseaux & 3241 & 153165 & 102178 & 91137 & 349721 \\
\hline & 2- Répartition du personnel entre les services (\%) \\
\hline $\begin{array}{l}\text { Chemins de fer } \\
\text { secondaires }\end{array}$ & 5,4 & 36,4 & 25,4 & 32,8 & 100 \\
\hline Grands réseaux & 0,9 & 43,9 & 29,2 & 26 & 100 \\
\hline \multicolumn{5}{|c|}{ 3- Nom bre d'agents pour 10 kilomètres } \\
\hline $\begin{array}{l}\text { Chemins de fer } \\
\text { secondaires }\end{array}$ & 0,8 & 5,6 & 3,9 & 5,4 & 14,7 \\
\hline Grands réseaux & 0,8 & 37,7 & 25 & 22,4 & 85,9 \\
\hline
\end{tabular}

La division Matériel et Traction des compagnies secondaires est dépassée en effectif par celle Voie et Bâtiments, situation inverse sur les grands réseaux. Les réseaux de chemins de fer secondaires connaissant une grande extension à partir de 1890, en 1911, on l'a dit, le matériel est en grande partie récent et cela suppose un entretien moindre que sur les lignes des grands réseaux où circulent encore des véhicules dont la vétusté était certaine ; en outre les faibles vitesses en usage sur les chemins de fer secondaires limitaient les contraintes imposées pour la surveillance du parc de locomotives, voitures et wagons.

La comparaison entre les nombres d'Ag/10 km de chaque service sur les deux types de réseaux est saisissante : en exceptant l'administration centrale, les grands réseaux emploient en moyenne six fois plus d'agents que les compagnies secondaires, les rapports sont d'environ sept pour Mouvement et Trafic, six pour Matériel et Traction, cinq pour Voie et Bâtiments (tabl. 53). Les frais de surveillance et d'entretien des voies sur 
les réseaux secondaires sont évalués au tiers du montant consenti par les grands réseaux pour leurs lignes à faible trafic. Les compagnies secondaires restreignent les dépenses de personnel dans les gares, les trains, les ateliers et les dépôts quand ils existent ; elles ne sont pas tenues au gardiennage des passages à niveau, elles sont libérées de contraintes d'exploitation, telles que le nombre de circulations quotidiennes ou de classes pour les trains de voyageurs; elles peuvent adapter l'offre à la demande, employer des engins de traction dont on obtient le meilleur rendement énergétique. On comprend les demandes réitérées des grands réseaux de bénéficier, hors leurs lignes maitresses, des libertés accordées aux compagnies secondaires ; une réponse positive de l'État aurait permis d'éviter, ou au moins de limiter, les déficits enregistrés notamment sur les lignes du plan Freycinet, souvent l'année même de leur mise en service ; le refus a été persistant.

Suivant les milieux desservis, le volume et la nature des trafics attendus différaient beaucoup : trafic industriel ou agricole, rural, périurbain ou touristique. À chaque cas correspondaient ses besoins spécifiques en hommes et moyens matériels.

Les compagnies acquièrent le matériel et recrutent le personnel nécessaires à l'exploitation de leur première ligne ; elles renforcent leurs moyens si le trafic dépasse les prévisions. Cette politique était surtout dictée par la multiplication des liaisons ; elle était dominée par le souci d'économiser ; aussi privilégiait-on l'amélioration de la productivité des personnels et du matériel. Une faible augmentation des moyens permettait de faire face au prolongement d'une ligne, même à l'ouverture d'une nouvelle ligne avec un terminus commun avec une liaison en service. Dans les vastes réseaux, le développement du parc et des effectifs d'employés s'imposait, mais sans perdre de vue la volonté de limiter les dépenses. Le problème des moyens est étroitement lié à celui de l'évolution des réseaux. 


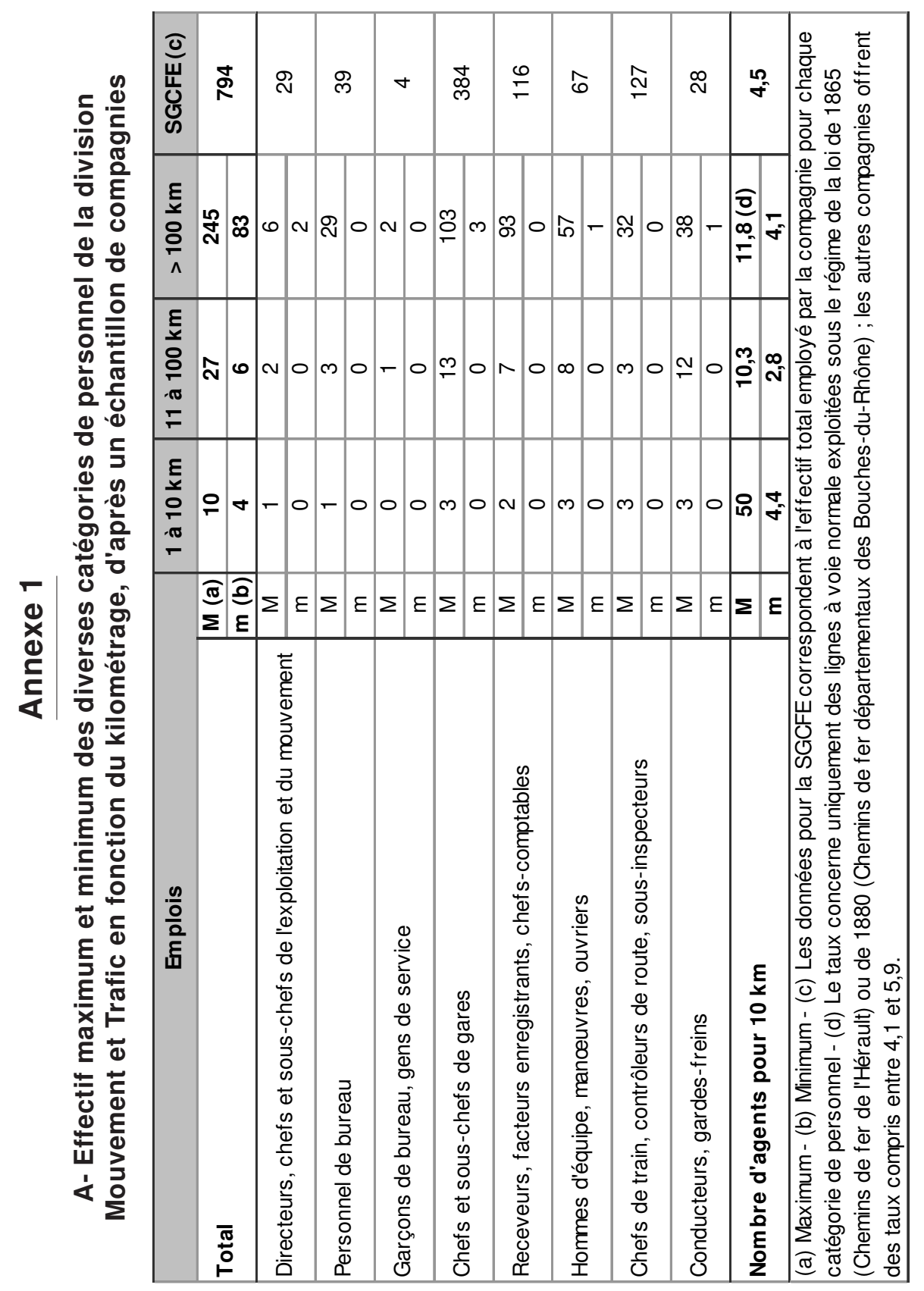




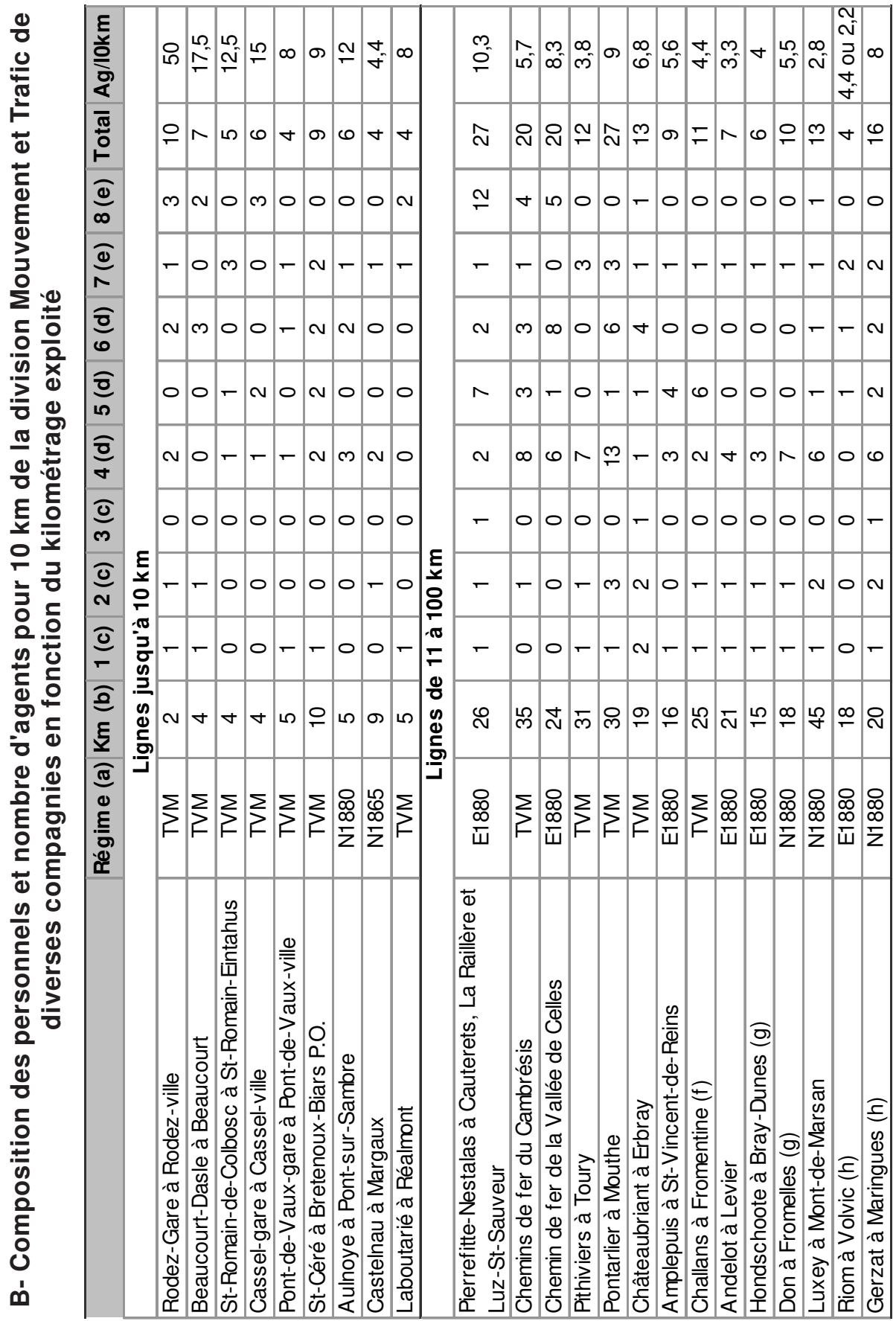

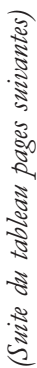




\begin{tabular}{|c|c|c|c|c|c|c|c|c|c|c|c|c|c|c|c|c|c|c|c|c|c|c|c|c|}
\hline$\frac{E}{\frac{5}{0}}$ & $\stackrel{\infty}{\stackrel{\infty}{\leftarrow}}$ & $\stackrel{\cong}{=}$ & F & 恶 & $\stackrel{\bullet}{\sim}$ & $\begin{array}{l}\infty \\
\omega\end{array}$ & טֶ. & ' & كا & $\dot{i}$ & 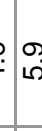 & $\dot{f}$ & $\underset{\sim}{\stackrel{N}{\sim}}$ & $\stackrel{m}{\sim}$ & & & 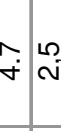 & 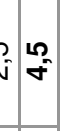 & & $\begin{array}{ll}0 \\
\dot{\sigma}\end{array}$ & & $\stackrel{8}{f} \sigma$ & $\overline{0}^{\circ}$ & ఝึ \\
\hline 푱 & $\stackrel{\text { L }}{\stackrel{\text { d }}{ }}$ & 요 & $\begin{array}{l}\mathscr{\infty} \\
\infty\end{array}$ & m్రా & $\stackrel{N}{\stackrel{N}{*}}$ & $\underset{\mathbb{N}}{\mathbb{N}}$ & $\stackrel{\swarrow}{\circ}$ & 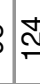 & 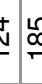 & $\begin{array}{lll}0 \\
0 \\
0\end{array}$ & $\underline{m}$ & $\bar{c}$ & m్ & $\infty$ & & $\stackrel{\circ}{\circ}$ & $\hat{\delta} \hat{m}$ & ১ & : & స్ N & & S & ষ্লি & ষ্ল \\
\hline$\infty_{\infty}^{\infty}$ & $\stackrel{\nabla}{\sim}$ & 0 & 으 & 0 & প্লি & $\widetilde{N}$ & - & $\stackrel{\infty}{m}$ & D c & 0 & $\curvearrowright$ & j & 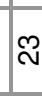 & $\infty$ & & $\stackrel{\circ}{ }$ & $\wedge \sim$ & v & $\begin{array}{l}\mathcal{O} \\
\bar{Z} \\
0 \\
\tilde{\sigma} \\
\sigma\end{array}$ & 0 & 00 & $\boldsymbol{D} \sim$ & $\bar{N}$ & $\stackrel{N}{N}$ \\
\hline () & $\stackrel{0}{ }$ & $\stackrel{N}{\sim}$ & $\stackrel{m}{7}$ & $\stackrel{10}{2}$ & ल & $\stackrel{\infty}{N}$ & \pm & 0 & 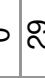 & $\vec{v} \tilde{\text { s }}$ & 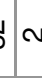 & $\sqrt{\simeq}$ & $\sim$ & 10 & & $\stackrel{\square}{\leftarrow}$ & 은 & $\stackrel{N}{\stackrel{N}{ }}$ & $\mid \begin{array}{l}0 \\
0 \\
0 \\
0\end{array}$ & $\ddot{~}$ & $\frac{10}{2} \overline{\text { in }}$ & $\pi$ & $\mathbb{N}$ & స్ల \\
\hline$\frac{\hat{0}}{0}$ & 음 & $\hat{n}$ & $\sim$ & ని & I & 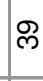 & $\infty$ & 7 & $=\curvearrowright$ & $\begin{array}{l}\vec{V} \\
\end{array}$ & $\sigma$ & ما & $\stackrel{\sim}{\sim}$ & $\infty$ & & & กิ $\sim$ & $\sqrt{0}$ & $\mid \begin{array}{l}\mathcal{E} \\
\mathbb{D} \\
\mathscr{D} \\
\mathcal{D} \\
\Phi\end{array}$ & 음 & ๓ & $\mathbf{v} ?$ & 寸 & ถో \\
\hline कृ & \& & ల్ల & 9 & $\stackrel{10}{2}$ & 0 & 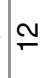 & \& & $\stackrel{2}{\circ}$ & र & $5=$ & $=\mathbb{N}$ & งి & 8 & $\forall$ & & 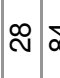 & ১ & $\stackrel{0}{\frac{0}{\sigma}}$ & $\begin{array}{c}\bar{\pi} \\
\bar{c} \\
\bar{z}\end{array}$ & 0 & $6 ?$ & $\mathbf{v}$ & $\underset{m}{m}$ & 悉 \\
\hline${ }_{\vec{\sigma}}^{\text {के }}$ & ட & 이 & 우 & مام & 음 & ৪ & $\mp$ & $m$ & 0 & $\triangle \widehat{\sigma}$ & $\vdots$ ○ & مص & $\underline{0}$ & $\stackrel{m}{-}$ & $\frac{\xi}{\underline{y}}$ & & $\frac{\Gamma}{m} \stackrel{m}{-}$ & ? & 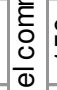 & 욤 & ले & 㔽 $\sigma$ & 8 & $\stackrel{2}{\wedge}$ \\
\hline (c) & $\tau$ & $\sim$ & 0 & - & - & - & - & -0 & $\supset \sim$ & $v-$ & -7 & $-N$ & - & - & $\begin{array}{l}8 \\
0 \\
0 \\
0\end{array}$ & $-c$ & $m 0$ & 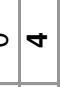 & 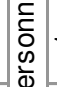 & - & 0 . & -0 & $\nabla$ & $\theta$ \\
\hline 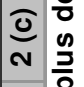 & $\stackrel{9}{-}$ & $\stackrel{N}{\sim}$ & 0 & 임 & $\stackrel{\bullet}{\circ}$ & ని & $\infty$ & 0 & $\supset$ & 8 & 7 & $=\bar{N}$ & $\sigma$ & $\wedge$ & 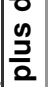 & & $\stackrel{\sim}{\sim} \sim$ & v & & $\therefore 0$ & $\infty \stackrel{\leftarrow}{\infty}$ & $\begin{array}{l}\boldsymbol{N} \\
\mathbf{n}\end{array}$ & - & $\tau$ \\
\hline $\begin{array}{l}0 \\
\tau \\
\tau\end{array}$ & 6 & $\nabla$ & L & ما & $\lambda$ & $m$ & m & $\sim$ & $v m$ & 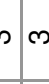 & $\sim$ & ת & m & $\nabla$ & $\mid \begin{array}{l}\vec{z} \\
\tilde{\pi} \\
: \overline{0} \\
0\end{array}$ & $0 \bar{c}$ & $\bar{\sim} \sim$ & $\checkmark \mathbb{N}$ & & m. & $-\sigma$ & o & 0 & $\Lambda$ \\
\hline 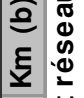 & 完 & $\stackrel{m}{m}$ & 오 & 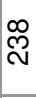 & 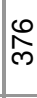 & $\begin{array}{l}\mathscr{D} \\
\infty\end{array}$ & $\stackrel{\infty}{-\infty}$ & 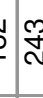 & 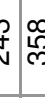 & : & ปి & $j \frac{\varphi}{\infty}$ & స్ల & के & \begin{tabular}{|l|}
$\mathbf{a}$ \\
$\mathbf{x}$ \\
0 \\
0 \\
0 \\
0
\end{tabular} & & 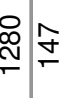 & 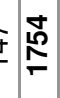 & N & 令) & 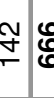 & $\stackrel{8}{\circ}$ & 号 & ஜ్రి \\
\hline 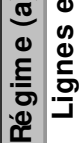 & $\begin{array}{l}\infty \\
0 \\
\frac{\infty}{z} \\
z\end{array}$ & $\begin{array}{l}\stackrel{8}{\infty} \\
\infty \\
\Sigma \\
\Sigma\end{array}$ & $\sum$ & $\sum$ & $\sum$ & $\sum$ & & $\begin{array}{l}\infty \\
\infty \\
\infty \\
\dot{u}\end{array}$ & $\sum^{\circ}$ & 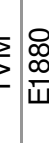 & $\begin{array}{l}\infty \\
\infty \\
\infty \\
\infty \\
w\end{array}$ & 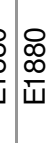 & & $\sum_{i}$ & 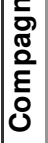 & & 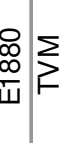 & 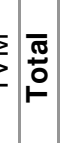 & 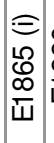 & & & & : & $\begin{array}{l}\bar{\pi} \\
\stackrel{0}{0} \\
-\end{array}$ \\
\hline & 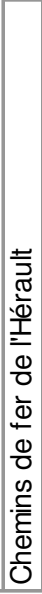 & 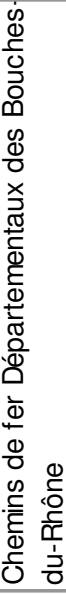 & 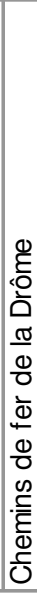 & 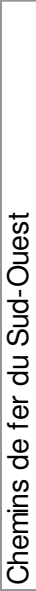 & 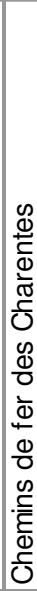 & 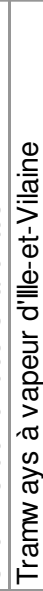 & 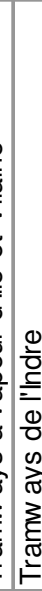 & 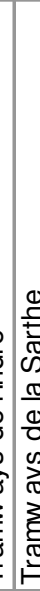 & 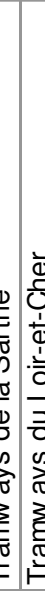 & 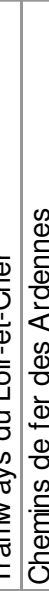 & 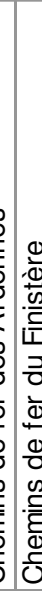 & 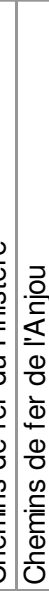 & 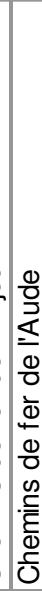 & 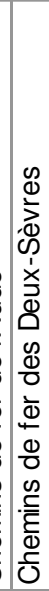 & & 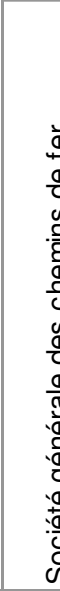 & 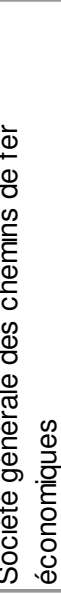 & & & 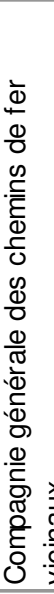 & & & 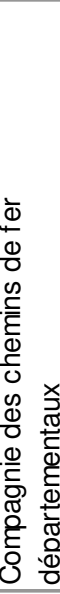 & \\
\hline
\end{tabular}




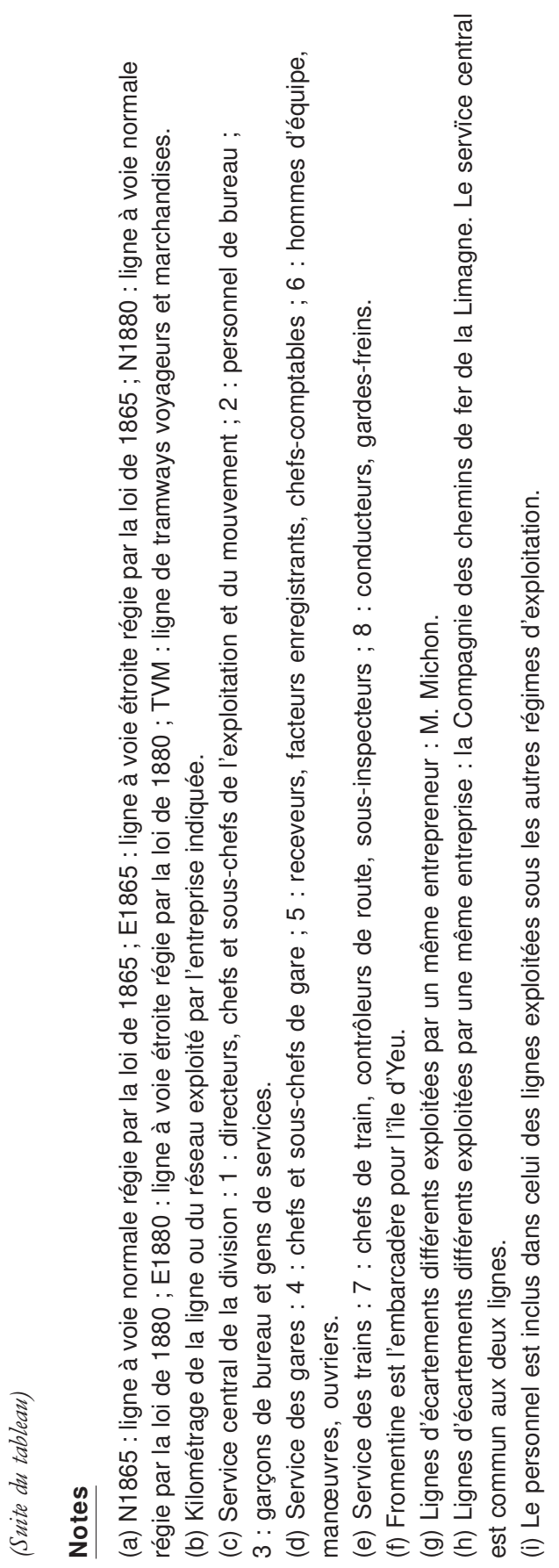




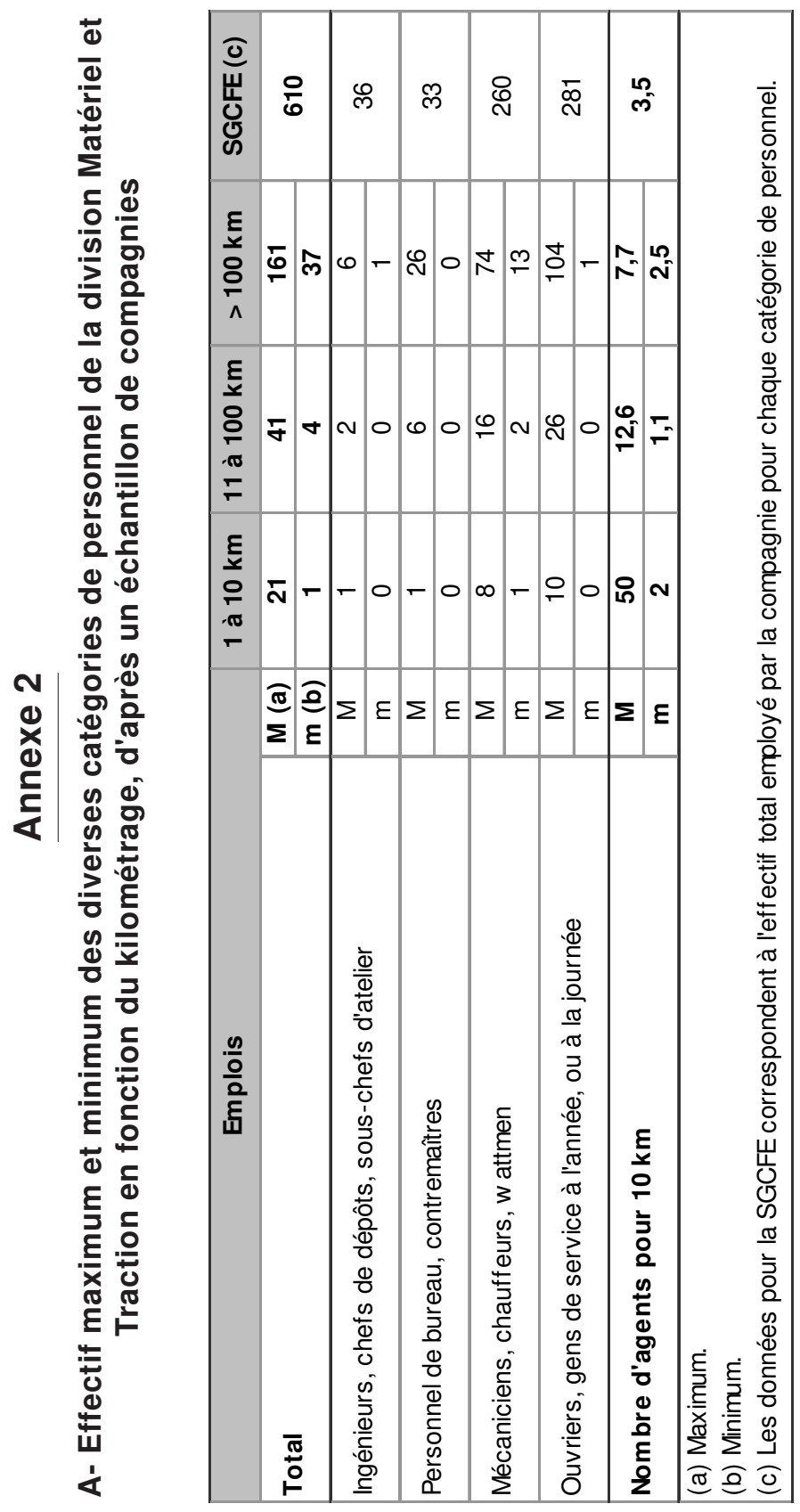




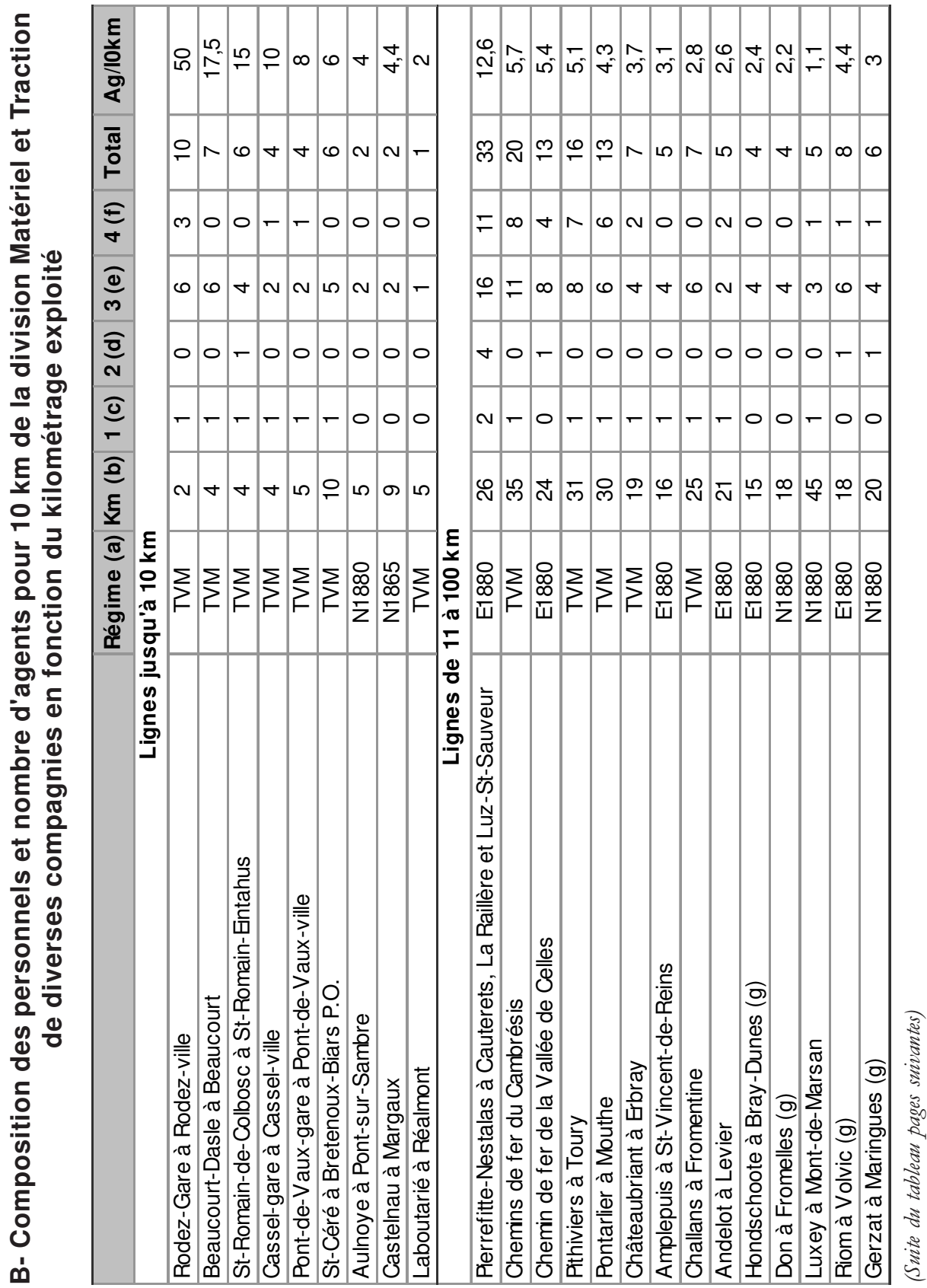




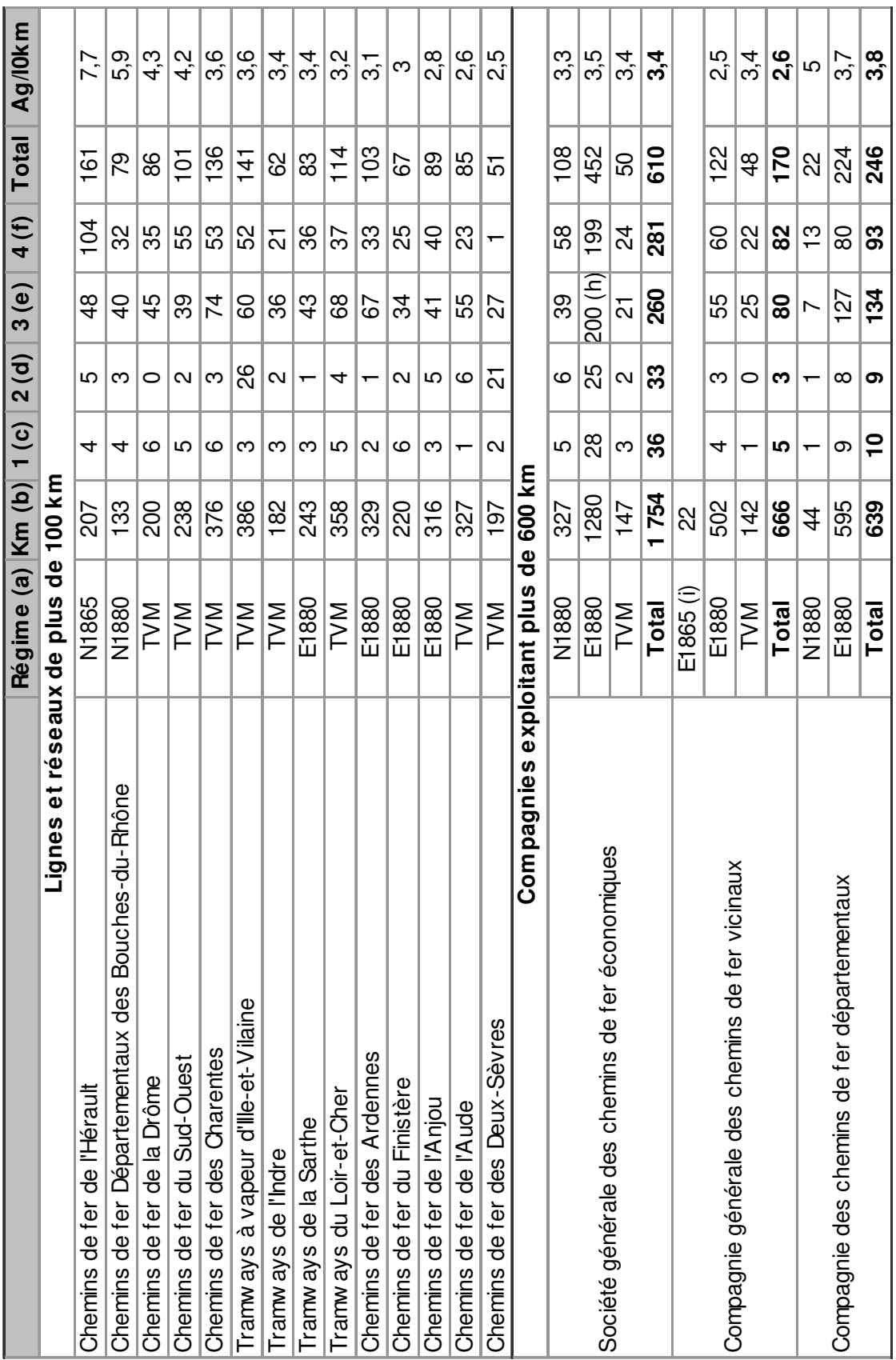




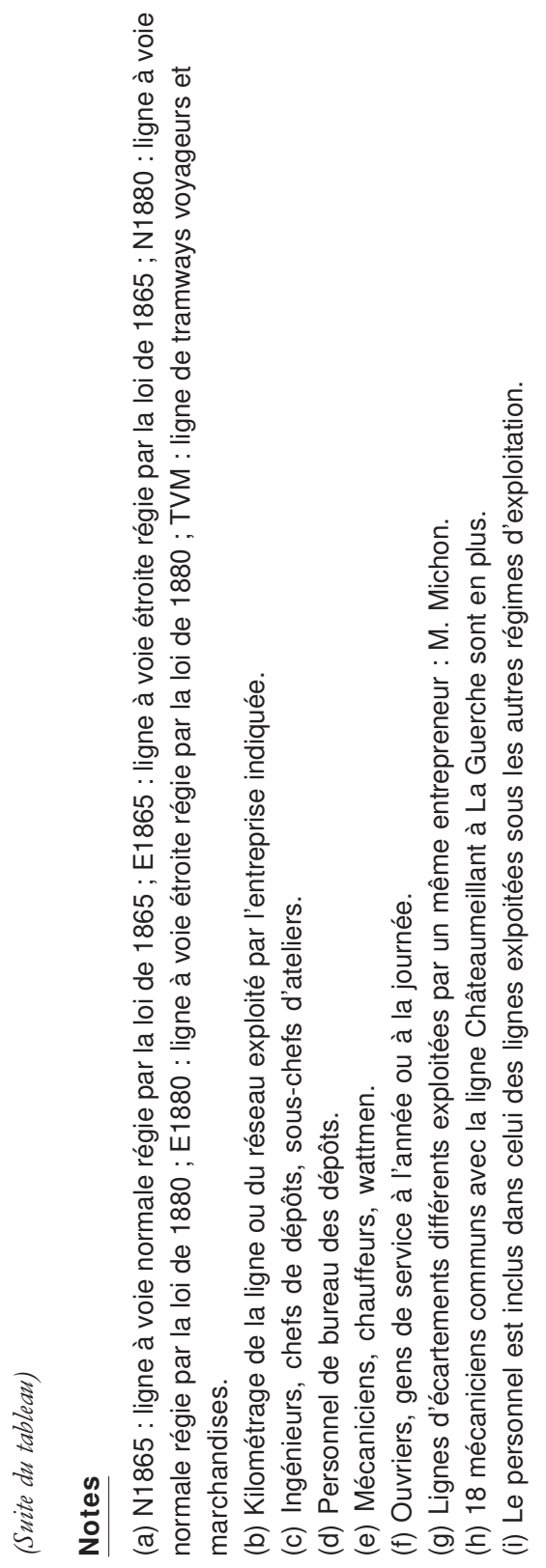




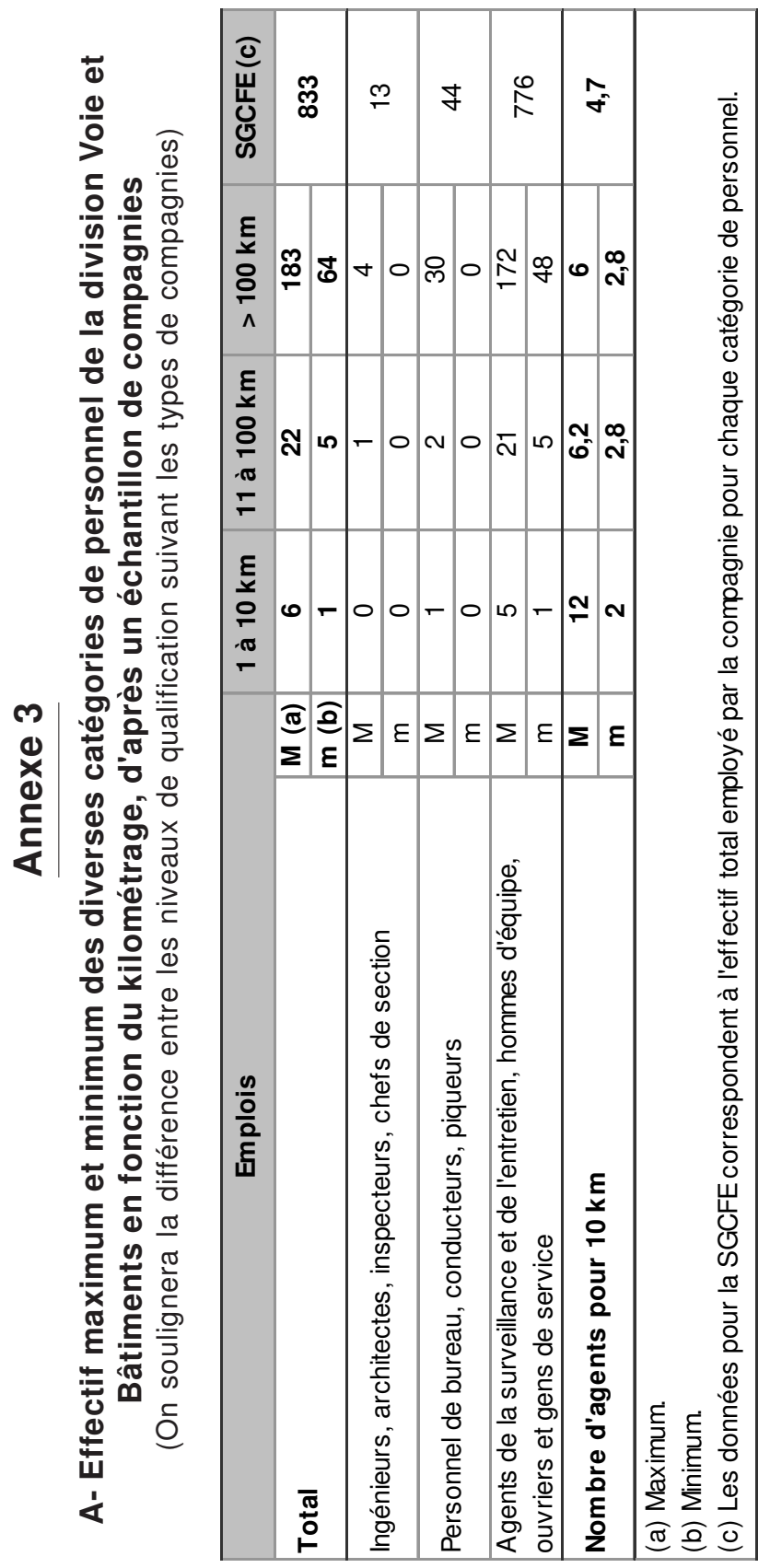




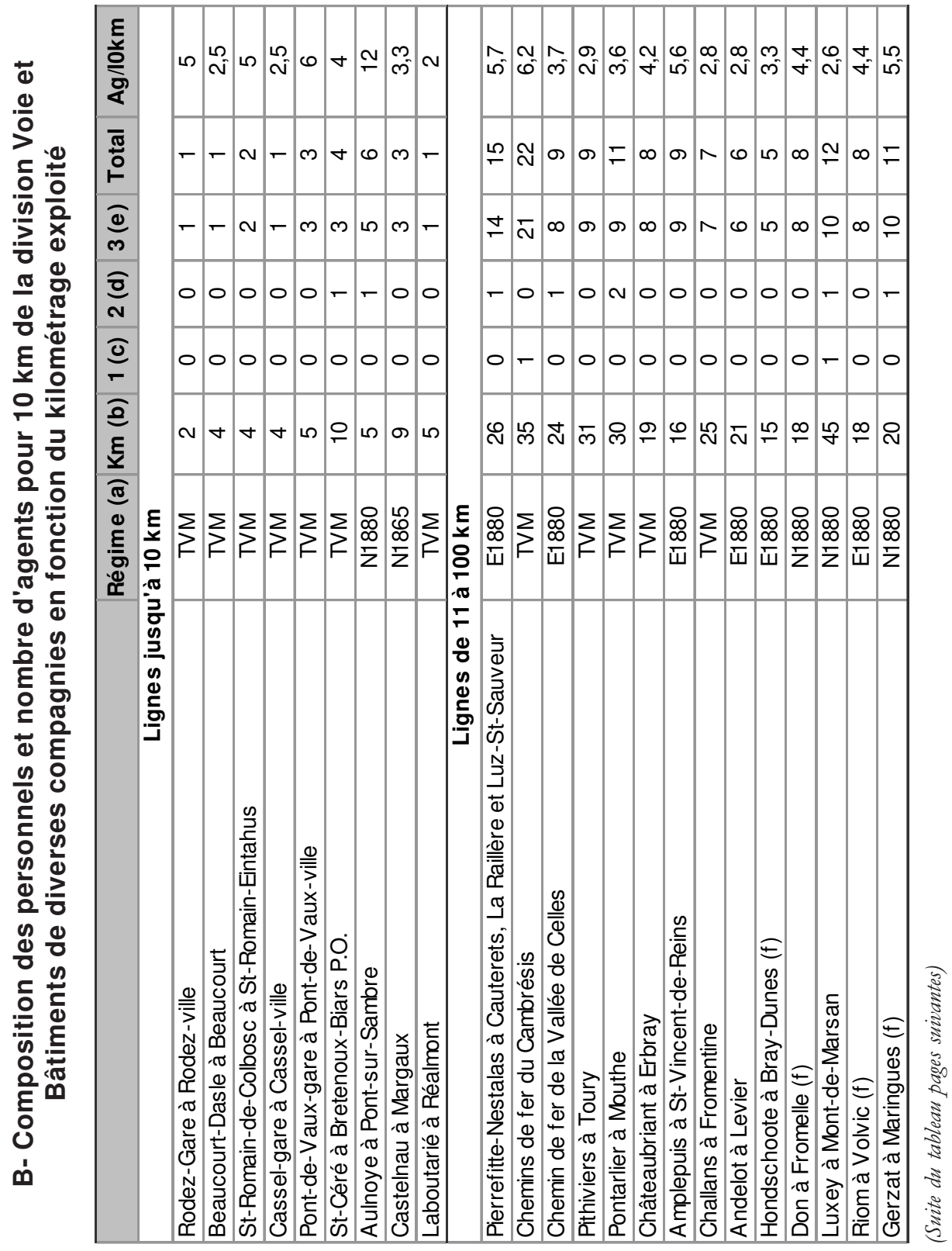




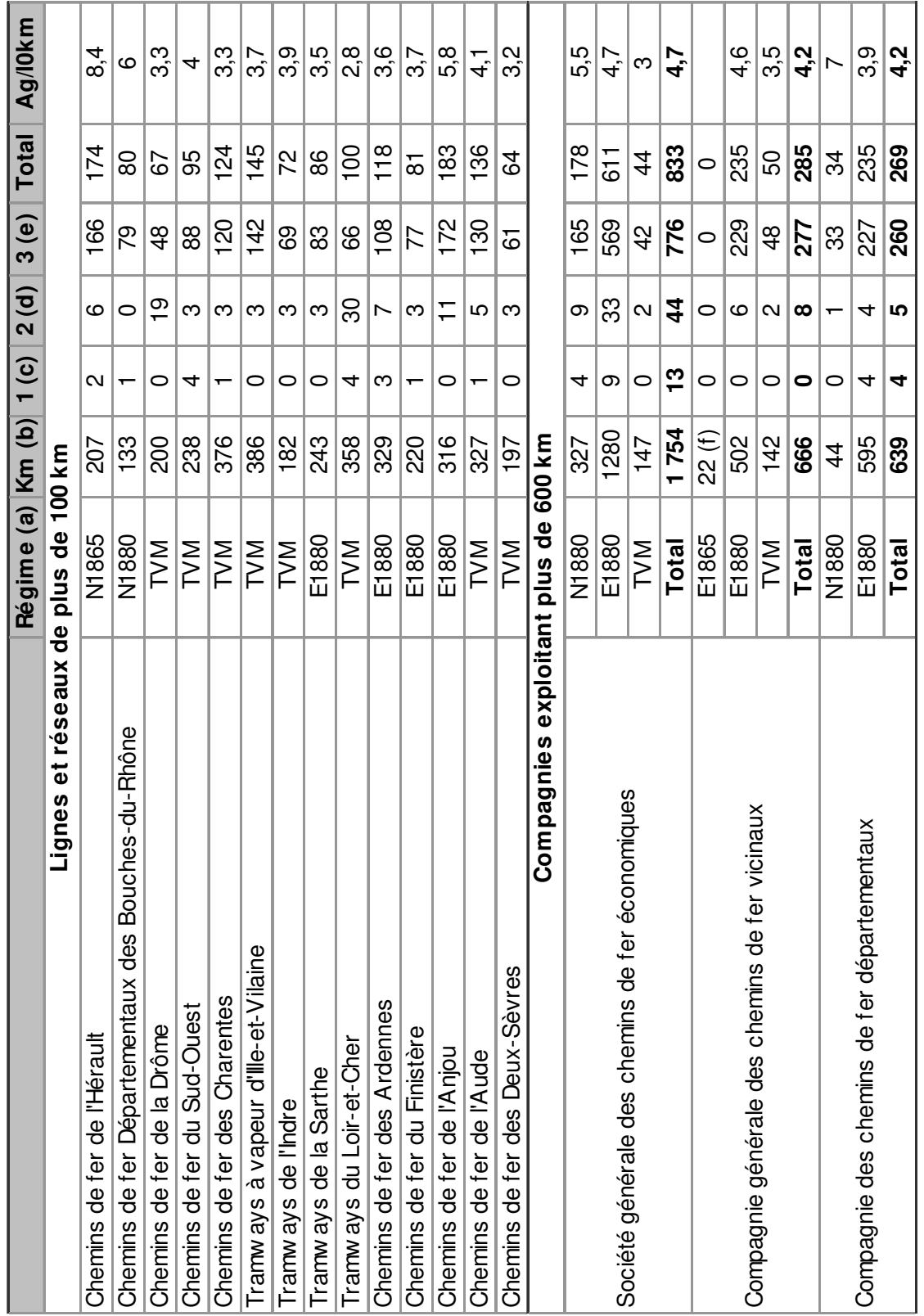




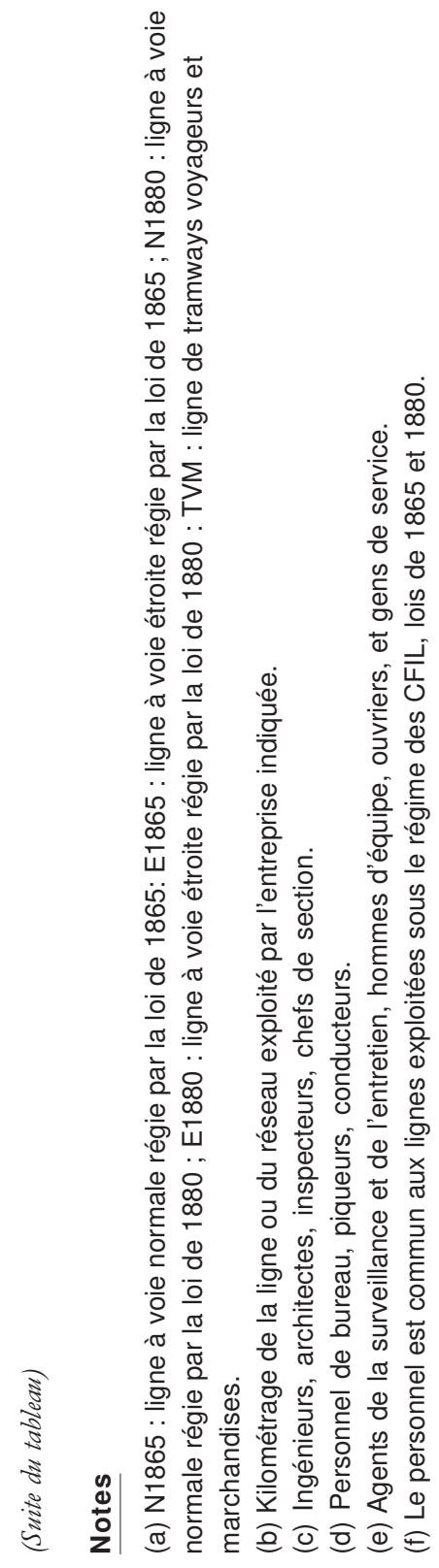




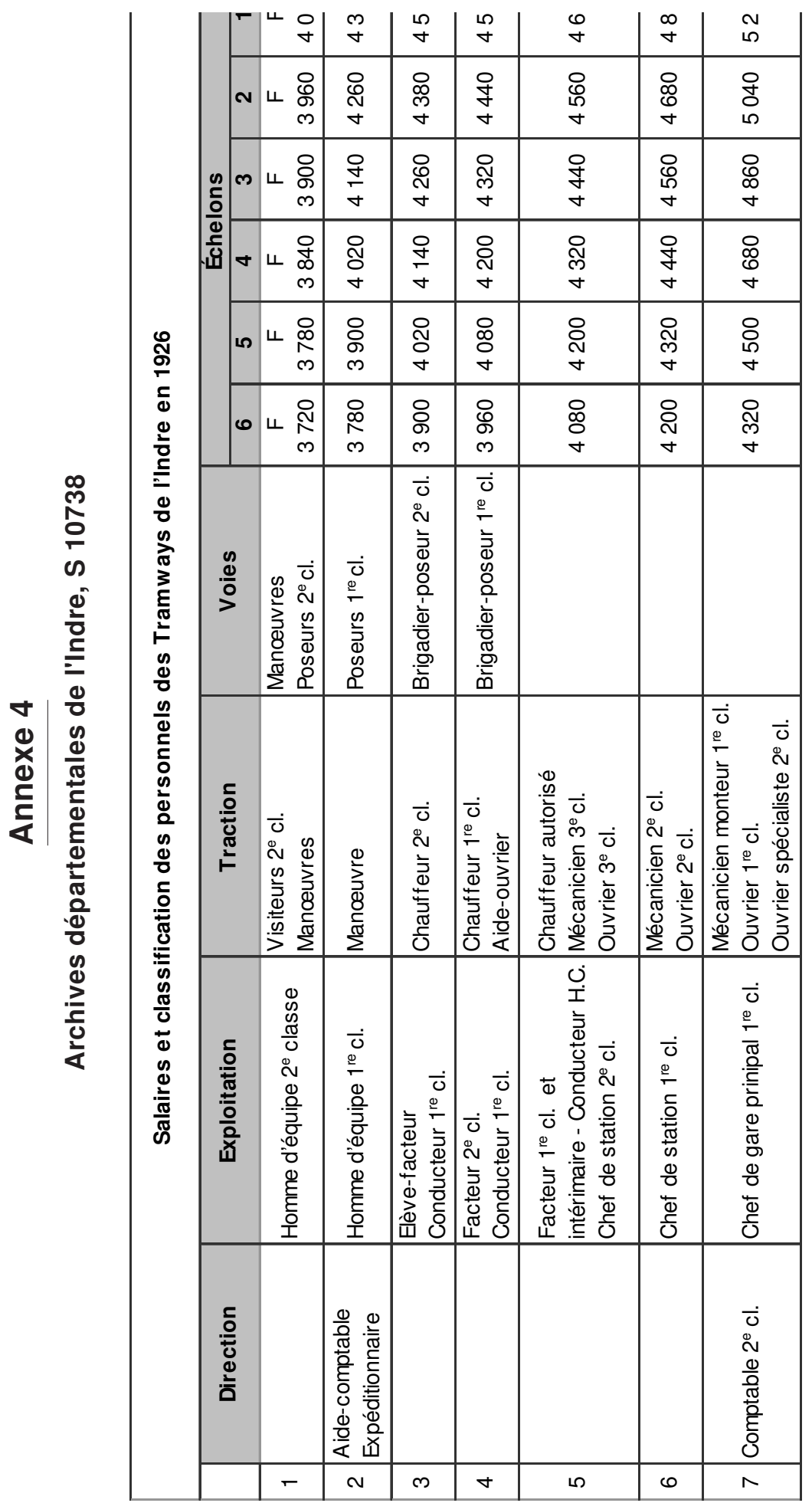




\begin{tabular}{|c|c|c|c|c|c|c|c|c|}
\hline \multirow{6}{*}{ 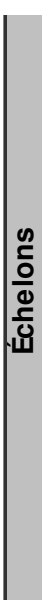 } & б & $\begin{array}{l}10 \\
10\end{array}$ & $\bar{\sigma}$ & ชَ & $\stackrel{0}{\wedge}$ & $\stackrel{m}{\wedge}$ & $\begin{array}{l}\text { in } \\
\infty\end{array}$ & \multirow{11}{*}{ 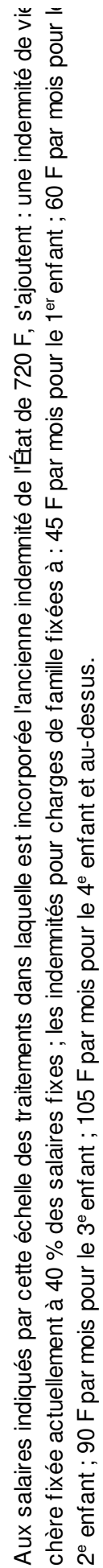 } \\
\hline & $\begin{array}{l}\stackrel{O}{\infty} \\
\stackrel{N}{ } \\
i\end{array}$ & $\begin{array}{l}\text { \& } \\
\text { + } \\
\text { L }\end{array}$ & $\begin{array}{l}0 \\
\infty \\
\infty \\
10\end{array}$ & $\begin{array}{l}\text { ㅁ } \\
\underset{0}{0}\end{array}$ & $\begin{array}{l}\stackrel{N}{N} \\
0\end{array}$ & 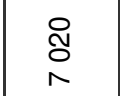 & $\begin{array}{l}\stackrel{\text { N }}{\infty} \\
\infty\end{array}$ & \\
\hline & $\frac{8}{10}$ & $\begin{array}{l}\stackrel{\text { N }}{\text { N}} \\
\text { in }\end{array}$ & $\begin{array}{l}\text { 웅 } \\
6 \\
\text { L }\end{array}$ & $\begin{array}{l}8 \\
8 \\
0\end{array}$ & 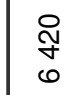 & 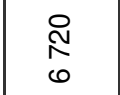 & $\begin{array}{l}\text { న } \\
\text { চ }\end{array}$ & \\
\hline & 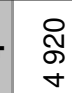 & $\begin{array}{l}\text { O } \\
\text { 10 }\end{array}$ & $\begin{array}{l}\text { o } \\
\text { L }\end{array}$ & $\begin{array}{l}8 \\
10 \\
10\end{array}$ & $\frac{\stackrel{\Upsilon}{0}}{0}$ & 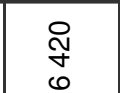 & $\begin{array}{l}\text { ণ্ণ } \\
N\end{array}$ & \\
\hline & $\underset{⿱}{\stackrel{O}{+}}$ & $\begin{array}{l}\bigotimes \\
\infty \\
\forall\end{array}$ & $\frac{8}{60}$ & $\begin{array}{l}\text { Nิ } \\
\text { గొ } \\
\text { L }\end{array}$ & $\begin{array}{l}\text { న } \\
\infty \\
10\end{array}$ & $\frac{\stackrel{\curvearrowright}{N}}{0}$ & $\begin{array}{l}\text { ণิ } \\
\text { N }\end{array}$ & \\
\hline & $\begin{array}{l}8 \\
\qquad \\
+\end{array}$ & 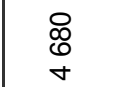 & 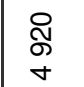 & 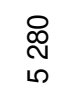 & $\begin{array}{l}\text { స్ } \\
\text { ஸి }\end{array}$ & $\begin{array}{l}\text { § } \\
\infty \\
10\end{array}$ & 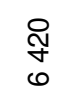 & \\
\hline 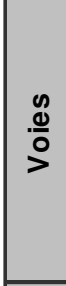 & 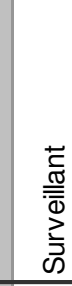 & 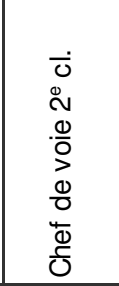 & 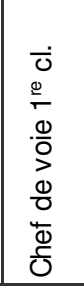 & & & & & \\
\hline 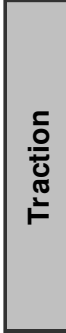 & 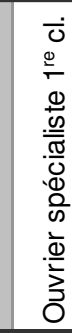 & 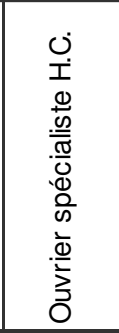 & 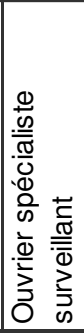 & 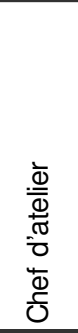 & 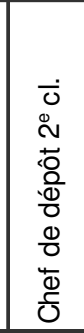 & 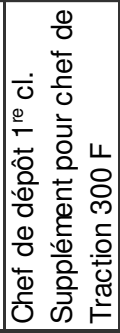 & & \\
\hline 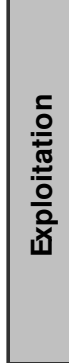 & 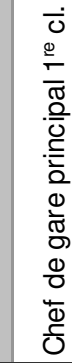 & 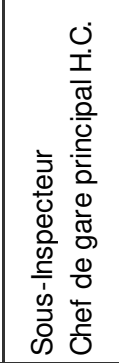 & 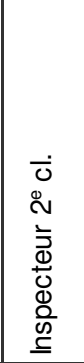 & & 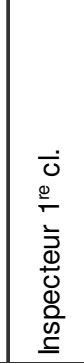 & 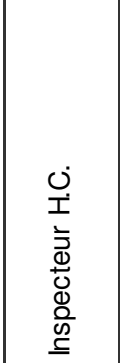 & 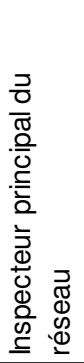 & \\
\hline 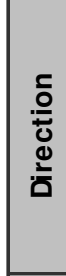 & & 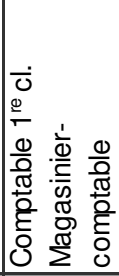 & & 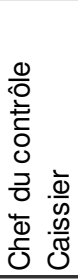 & 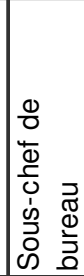 & 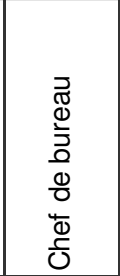 & 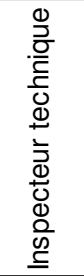 & \\
\hline & $\infty$ & $\sigma$ & 으 & $\mp$ & $\cong$ & $\stackrel{m}{\sim}$ & $\underset{\sim}{\nabla}$ & \\
\hline
\end{tabular}


